\title{
Review Article \\ Mechanism of Catch Force: Tethering of Thick and Thin Filaments by Twitchin
}

\author{
Thomas M. Butler and Marion J. Siegman \\ Department of Molecular Physiology and Biophysics, Jefferson Medical College, Thomas Jefferson University, Philadelphia, \\ PA 19107, USA \\ Correspondence should be addressed to Marion J. Siegman, marion.siegman@jefferson.edu
}

Received 20 January 2010; Accepted 10 March 2010

Academic Editor: Henk L. M. Granzier

Copyright ( $\odot 2010$ T. M. Butler and M. J. Siegman. This is an open access article distributed under the Creative Commons Attribution License, which permits unrestricted use, distribution, and reproduction in any medium, provided the original work is properly cited.

\begin{abstract}
Catch is a mechanical state occurring in some invertebrate smooth muscles characterized by high force maintenance and resistance to stretch during extremely slow relaxation. During catch, intracellular calcium is near basal concentration and myosin crossbridge cyctng rate is extremely slow. Catch force is relaxed by a protein kinase A-mediated phosphorylation of sites near the N- and $\mathrm{C}$ - temini of the minititin twitchin $(\sim 526 \mathrm{kDa})$. Some catch force maintenance car also occur together with cycling myosin crossbridges at submaximal calcium concentrations, but not when the muscle is maximally activated. Additionally, the link responsible for catch can adjust during shortening of submaximally activated muscles and maintain catch force at the new shorter length. Twitchin binds to both thick and thin filaments, and the thin filament binding shown by both the $\mathrm{N}$ - and Cterminal portions of twitchin is decreased by phosphorylation of the sites that regulate catch. The data suggest that the twitchin molecule itselfis the catch force beanng tether between thick and thin filaments. We present a model for the regulation of catch in which the twitchin tether can be displaced from thin filaments by both (a) the phosphorylation of twitchin and (b) the attachment ofhigh force myosin crossbridges.
\end{abstract}

\section{Introduction}

The hallmark of the contractile process in smooth muscle is its ability to maintain force with a very high economy, that is, a low expenditure of energy, through the slow cycling of force-bearing crossbridges. In certain invertebrate smooth muscles, such as adductors, force can be maintained for many hours. This force, familiar to those who have attempted to open the shells of oysters, scallops, clams, and mussels, as well as the contraction of the anterior byssus retractor muscle (ABRM) of the edible mussel Mytilus edulis, reflects an unusual contractile state called "catch." Functionally, the catch state is an adaptation that allows the muscle to resist stretch; this is important in the scallop, for example, in controlling gape (openness of its shell) and for the mussel in holding its byssus threads, which anchor it to rocks and other substrates, tautly. The term "catch" was coined nearly one hundred years ago by von Uexkull to describe the state of prolonged tonic force maintenance on the assumption that the muscle is "caught" in the shortened state by a ratchet mechanism [1]. In molluscan smooth muscle, calcium activates contraction by direct binding to myosin [2]. Upon cholinergic nerve stimulation of the intact $A B R M$, intracellular calcium rises rapidly and then decays to near-resting concentrations [3] (Figure 1(b)). It is at these low calcium concentrations that the catch state ensues, and (in the absence of stimulation) force declines very slowly, over a period of minutes, or even hours. Catch is characterized by an extremely slow relaxation of force and a very high resistance to extension in the face of a very low expenditure of energy $[4,5]$. Stimulation of serotonergic nerves "releases" catch, that is, causes rapid relaxation, which is mediated by the resultant increase in cAMP and activation of protein kinase A $[6,7]$. The simultaneous stimulation of excitatory and inhibitory nerves leads to a phasic contraction (Figure 1(a)), reflecting the overriding influence of some cAMP-dependent process, leading to decreased force production. The question of how cAMP 


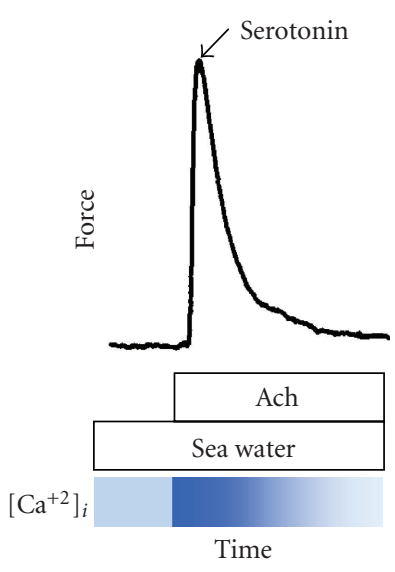

(a)

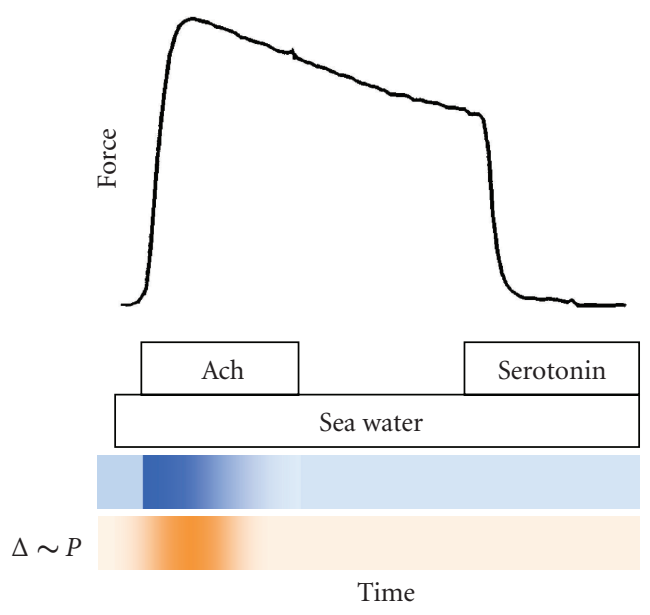

(b)

FIGURE 1: Contractile responses of intact anterior byssus retractor muscle of Mytilus edulis. Muscles were bathed in artificial seawater (ASW) and then treated as shown. (a) Phasic contraction. Muscle was activated with acetylcholine ( $50 \mu \mathrm{M})$ and at peak force serotonin (10 $\mu \mathrm{M})$ was added. (b) Activation, catch, and release of catch in intact muscle. Muscle was activated with acetylcholine ( $50 \mu \mathrm{M})$ for 1.5 minutes which was washed out with ASW for 1.5 minutes. The condition of high-force maintenance is catch. Catch force was rapidly released upon addition of $10 \mu \mathrm{M}$ serotonin. Lower panel (orange) shows the time course of high-energy phosphate usage during activation and catch [8]. Lower panels (blue) show the time course of the accompanying changes in intracellular $\mathrm{Ca}^{++}$during phasic and catch contractions based on the work of Ishii et al. [3].

leads to abrupt relaxation was of interest to us because it might provide a handle on how highly economical force (latch, catch) was maintained and eventually released during relaxation in mammalian as well as invertebrate smooth muscles.

\section{Theories on the Mechanism of Catch}

Two main theories have been put forward for the catch mechanism (reviewed by Ruegg [9]). The "linkage hypothesis", proposed by Lowy et al. [10], suggests that the links between actin and myosin filaments (myosin crossbridges) cycle rapidly during the early, phasic portion of contraction, but are either locked in the attached state or detach very slowly during the catch phase. This stands in contrast to the "paramyosin hypothesis," proposed earlier by Ruegg [11], which assumes the operation of two functionally distinct linkage systems for contraction and catch. In this model, myosin crossbridges link with actin, but catch linkages may involve paramyosin-paramyosin interactions among neighboring thick filaments.

Catch muscles of molluscs contain actin filaments that are generally similar to those in other invertebrate muscles as well as vertebrate muscles (see extensive review in [12]). However, the thick filaments are very large, with diameters of $40-90 \mathrm{~nm}$ and lengths up to $50 \mu \mathrm{m}$ [10]. The thick filaments are comprised of paramyosin, which forms a large diameter core, and a monolayer of myosin which covers the surface [13]. Paramyosin serves as a scaffold for myosin; it is bipolar and myosin that attaches to it in a helical antipolar manner [14]. The paramyosin content of the filaments varies, being largest in muscles generating high forces. Thick filaments in invertebrate muscles generating high forces also typically have the largest diameters and longest lengths [15]. Force produced by the intact ABRM is about $10-14 \mathrm{~kg} \cdot \mathrm{wt} / \mathrm{cm}^{2}$, in contrast to vertebrate skeletal muscle, where the force is $\sim 2.3 \mathrm{~kg} \cdot \mathrm{wt} / \mathrm{cm}^{2}[10,16]$. Thick filaments from catch muscles also contain the protein catchin (myorod), which is an alternative product of the myosin heavy chain gene that has a unique $\mathrm{N}$-terminal sequence of 156 residues and the Cterminal 830 residues of the myosin heavy chain $[17,18]$. The function of catchin is not known. In in vitro motility studies catchin was not essential for demonstration of the catch state [19]. Although there is some ultrastructural evidence for aggregation of thick filaments in catch [20], other studies showed the same filament distribution during phasic and catch contractions of ABRM and provided compelling evidence that filament aggregation may be a fixation artifact [21]. In more recent studies by Takahashi et al. [22] no thick filament aggregation was observed in the cross-section of the fibers quickly frozen in the relaxed, actively contracting and in the catch state. The thick filaments were occasionally interconnected with each other either directly or by distinct projections in all the three states studied, but to the greatest extent in the catch state, leading the authors to suggest that thick filament interconnections are responsible for the catch state. However, it is difficult to envision how links among thick filaments alone would maintain catch force. It is interesting that in vitro the actin-activated myosin ATPase activity of catch muscle can be inhibited by disorderly aggregated "amorphous" paramyosin [23], but not by the orderly aggregated paramyosin core of thick filaments [24]. Ruegg [25] speculated that such a phase change within 
paramyosin filaments may be induced by phosphorylation of paramyosin by a cAMP-dependent protein kinase [26, 27]. This could influence the interaction of paramyosinmyosin and/or paramyosin-paramyosin, actomyosin ATPase and possibly crossbridge movement, and thereby enhance catch [13]. The precise role of paramyosin in the contractile process of intact muscle remains an enigma.

There is evidence from other early studies that address the issues of relative crossbridge cycling rates during phasic and catch contractions, and the basis of the high resistance to stretch during catch. Sinusoidal vibrations of appropriate frequency and amplitude have been found to reduce force production during phasic contractions of vertebrate vascular smooth muscle as well as ABRM, with complete recovery of active force after cessation of vibration. However, during catch in ABRM, force fails to recover following the vibration-induced inhibition. The vibrations presumably act by increasing the rate of detachment of crossbridges [28, 29] and, if so, this would suggest that once detached, the crossbridges in catch do not reattach, or when reattached, do not generate force. On the other hand, Kobayashi et al. [30] found that sinusoidal vibrations reduced force but not stiffness during catch and argued that catch force may not simply be maintained by "locked-on" actin-myosin cross-links, as originally proposed by Lowy and Millman [31]. Along these lines, in a rather limited study, a higher stiffness to tension ratio was observed during catch than in active contraction [32]. Taken together with the observation of an exceptionally high, serotonin-sensitive resistance to stretch during catch, it is conceivable that some additional filament interactions occur during catch. During catch, the muscle shows an extremely high resistance to stretch (up to $100 \mathrm{~kg} / \mathrm{cm}^{2}$ ) but does not redevelop force following a quick release; upon return to the initial length, the prerelease force is restored $[16,31,33]$. Catch stiffness is similar to rigor of skeletal muscle, but unlike rigor, is not due to the depletion of ATP from the muscle and is reversible [5, 34]. This is in contrast to the phasic portion of the contraction, in which the muscle resists stretch and redevelops force following a quick release of $5 \%-10 \%$ of the muscle length $[16,31]$. When catch is released, the resistance to stretch decreases, with no compromise of the phasic contractile response [35].

\section{Catch and the Economy of Force Maintenance}

Although initiated and controlled by neural activity, the cellular basis of phasic and tonic behavior in invertebrate smooth muscles, like mammalian muscles, resides in the contractile machinery itself. An important property that these smooth muscles share is the ability to regulate the energy cost of force production and force maintenance. The ability to maintain high force with low-energy usage was first noted from measurements of heat production during the contraction of the pharynx muscle of the edible snail (Helix pomatia) by Bozler in 1930 [36]. Later measurements of oxygen consumption during contraction of the ABRM of Mytilus edulis showed that the energy usage for maintaining a given force during catch is about one-tenth of that required during the preceding period of contraction $[4,5]$. Due to their ability to maintain force with little energy usage (actomyosin ATPase activity), vertebrate and invertebrate smooth muscles are considered to be very "economical". The marked change in economy was traced to an ability to regulate the rate at which crossbridges cycle during the course of a contraction, such that crossbridge cycling rate was high during force development and very slow during force maintenance $[16,37-39]$. Early evidence of a slowcycling rate and a catch-like state during force maintenance (based on the absence of force recovery following a quick release) in rabbit main pulmonary artery was reported in a discussion of catch by the Somlyos [40]. The condition of highly economical force maintenance in invertebrate smooth muscle later served as the model for what is known as the "latch" state of mammalian smooth muscle [39], primarily because of similarities in mechanical behavior (slow velocity of shortening) of the two muscle groups. At that time there was no information on the regulation of catch.

\section{Studies on Permeabilized Muscles}

Phasic and catch contractions have been studied in muscles from ABRM that have been permeabilized by treatment with detergents or by glycerol extraction. Such preparations provide the investigator the advantages of control of the milieu of a structured contractile system and, as such, a model system that is useful for the study of the regulation of catch. The addition of calcium $(10 \mu \mathrm{M})$ fully activates contraction by binding to myosin [41], and its removal establishes the catch state [42]. Catch can be released by the addition of cyclic AMP or by addition of the catalytic subunit of the cyclic AMP-dependent kinase [32, 43-46], which has no effect on force development. Guth and colleagues [47] measured the ATPase activity of permeabilized ABRM of Mytilus edulis and found that following a calcium-induced contraction lowering the calcium concentration rapidly reduced the ATPase activity to resting levels at a time when catch force had declined by only $30 \%-50 \%$ of maximum. Treatment with cAMP released catch force with no effect on ATPase activity. In a parallel experiment on the guinea pig taenia coli, the changes in force and corresponding ATPase activity during the application and subsequent removal of calcium were qualitatively similar to those of the ABRM, including a period of slow relaxation, during which Pi rather than cAMP was used to released the latch state and accelerate relaxation (similar responses, although probably based on very different mechanisms). The taenia coli, like the ABRM, shows no recovery of force following a quick release in length during the period of slow relaxation [48]. Thus, the permeabilized muscles faithfully follow the behavior of intact catch and mammalian smooth muscles, and the two muscle groups are strikingly similar in their contractile behavior.

The role of cAMP with the activation of the cAMPdependent kinase in the release of catch is certain [32, 43, 46, 49]. Paramyosin, myosin rod, and myosin light chains have been shown to be substrates for phosphorylation. Watabe et al. [50] noted that in myofibrils isolated from the 
white adductor (catch) muscle of Mercenaria, thiophosphorylation of paramyosin, through the action of an endogenous kinase resulted in an inhibition of ATPase activity. One of the regulatory light chains of myosin of scallop adductor muscle has been shown to be phosphorylated by a calcium- and calmodulin-independent cAMP-dependent protein kinase $[51,52]$. The kinase also phosphorylated myosin heavy chains and paramyosin [53]. Phosphorylatable serine residues close to the C-terminus on the rod portion of myosin have been identified, and this favors folding (6 S-10 S transition) of the myosin molecule $[46,54,55]$.

Castellani and Cohen showed that phosphatase inhibition with $\mathrm{NaF}$, or the use of a nonhydrolysable substrate such as ATP- $\gamma$-S prevents catch, but not the initial force development [46]. This suggests that calcium and phosphorylation of specific proteins (either kinases or contractile proteins) have separate roles in the regulation of contraction and catch, but these have not yet been defined. They also showed that prolonged treatment of the muscle with detergent during permeabilization leads to the loss of catch force maintenance which can be restored by the addition of calcineurin, a calcium-calmodulin-regulated type 2B phosphatase [56]. Further, trifluoroperazine, a calmodulin inhibitor, reversibly accelerates the loss of catch force. Taken together, the results point to a calcium-regulated phosphatase, possibly activated during the onset of contraction, which is essential for the maintenance of catch force [56]. These two studies provided the first link between the phosphorylation of some substrate to release catch, and the calcium-dependent dephosphorylation of that substrate during the transition to catch following activation. It is not known, however, whether more than one kinase operates and how this relates to the phosphatases that have been implicated, nor is there information on the dephosphorylation reactions. Traditional studies measuring ${ }^{32} P$ incorporation into various proteins from $\gamma^{32} P$-ATP were not successful at identifying the proteins whose change in state of phosphorylation mediate entry into and/or exit from catch [57].

\section{Phosphorylation of a High Molecular Weight Protein Regulates Catch}

In order to identify the target(s) of A kinase-mediated phosphorylation, we set out to determine the proteins that undergo a change in phosphorylation on a time course that corresponds to the release of catch in permeabilized ABRM [58]. To do this, we took advantage of technology involving flash photolysis of caged compounds. Specifically, permeabilized muscles were put into catch in the presence of ATP and caged ${ }^{33} P$-ATP. cAMP was then added and the muscles were immediately subjected to a UV flash that caused photolysis of the caged ATP and a step increase in the ${ }^{33} P$ content of ATP. The muscles were frozen at different times after the flash. Figure 2 shows that there was a large incorporation of ${ }^{33} \mathrm{P}$ into a high-molecular-weight protein (about $600 \mathrm{kDa}$ ) during the time that the catch force underwent relaxation. Surprisingly, this could also be shown in muscles that were not subjected to flash photolysis and

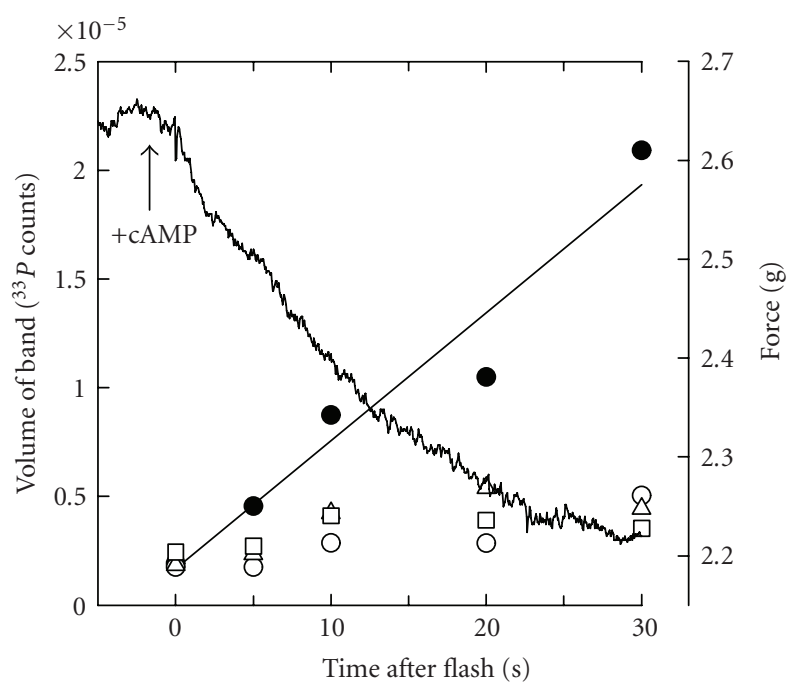

FIGURE 2: Time course of release of catch force and corresponding incorporation of ${ }^{33} \mathrm{P}$ into proteins of permeabilized ABRM following cAMP treatment and photolysis of caged ${ }^{33} P$-ATP. Muscles were frozen at times indicated after photolysis. Labeled proteins are shown by the following symbols: $\square, \sim 45 \mathrm{kDa} \bigcirc, \sim 100 \mathrm{kDa} ; \Delta, \sim$ $200 \mathrm{kDa} ; \bullet$, high-molecular-weight protein. $T=20 \mathrm{C}$. Reproduced from [58] with permission.

were, rather, simply incubated in ${ }^{32} \mathrm{P}$-ATP before addition of cAMP. A phosphorimage of a gel-containing proteins from muscles incubated in ${ }^{32} \mathrm{P}$-ATP and frozen under different mechanical conditions is shown in Figure 3. The only protein showing an increased degree of phosphorylation with the addition of cAMP was the high-molecular-weight protein. The only other obvious change in ${ }^{32} \mathrm{P}$ incorporation in the different conditions was an increase in phosphorylation in the myosin light chain region of the gel with an increase in calcium concentration (compare lanes A and B). Importantly, this did not increase further with cAMP treatment, and the amount of ${ }^{32} \mathrm{P}$ incorporated was only about $13 \%$ of that in the high molecular weight protein. We also found that (1) inhibition of the cAMP-dependent protein kinase, which prevented the cAMP-induced increase in phosphorylation of the protein, also inhibited the cAMP-dependent release of catch; (2) cAMP dependent thiophosphorylation of the protein prevents catch; (3) in intact muscle the phosphorylation of the protein is low when the muscle is activated by acetylcholine, but increases significantly when catch is released with serotonin treatment.

\section{The High Molecular Weight Protein Whose Phosphorylation State Regulates Catch is Twitchin}

The molecular weight of the catch-regulating protein was similar to that of the minititin, twitchin. Twitchin is a member of a family of giant protein kinases, which also includes projectin and titin, having molecular masses from 700 to $>3000 \mathrm{kDa}$. Twitchin is a protein that is encoded by the gene $u n c-22$ in $C$. elegans, and gained its name 

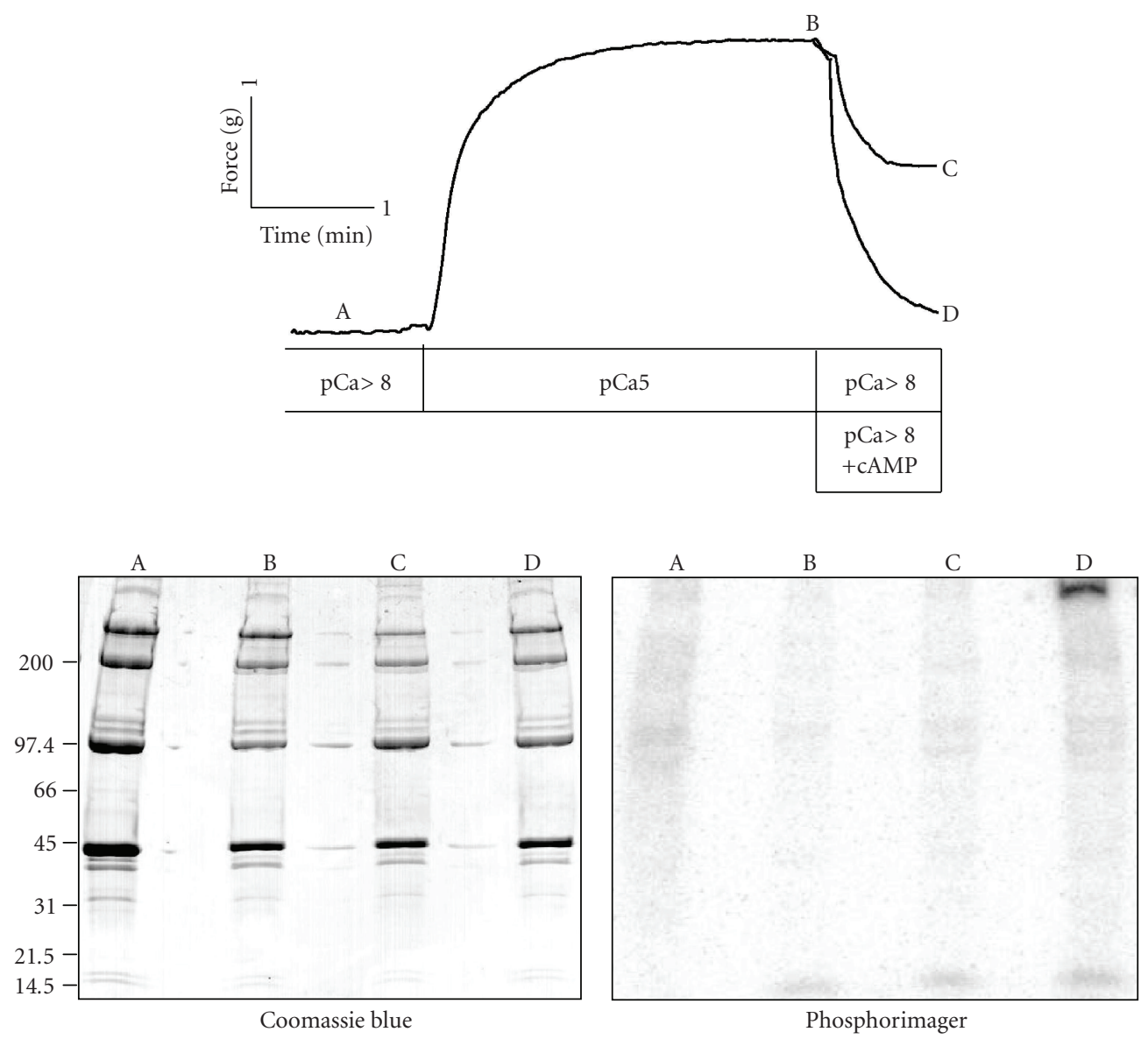

FIGURE 3: Protein phosphorylation under different mechanical conditions in permeabilized ABRM. Muscles were incubated with ${ }^{32} P$-ATP starting in an initial relaxing solution and then either frozen (a) after $4 \mathrm{~min}$ in relaxing solution, (b) after 1 minute in relaxing solution and 3 minutes in activation solution (pCa5), (c) treatment as in (b), plus 1 minute in relaxing solution (catch), and (d) after treatment as in (b), plus $1 \mathrm{~min}$ in relaxing solution containing cAMP (release of catch). Shown are a Coomassie Blue-stained 4\%-15\% acrylamide gradient gel and corresponding phosphorimager autoradiogram. $T=20 \mathrm{C}$. Reproduced from [58] with permission.

because animals lacking unc-22 showed a nearly constant twitching rather than prolonged undulating contractions of the body muscles [59]. Numerous immunolocalization studies have shown that twitchin is associated with the A band of nematode striated muscle [60], molluscan fast striated muscle, and thick filaments of molluscan smooth (noncatch) and catch muscles [61]. Along these lines, in the noncatch accessory radula closer muscle of Aplysia, a correlation between the cAMP-dependent phosphorylation of twitchin and the rate of relaxation was found [62].

The above findings prompted the use of a procedure designed for twitchin to isolate and purify the highmolecular-weight protein from Mytilus ABRM. The mobility in gels of the protein isolated in this manner was identical to the catch-regulating protein, and it was phosphorylated by the catalytic subunit of protein kinase A. Primers designed from an N-terminal amino acid sequence of a peptide from the catch-regulating protein and from the sequence of Aplysia twitchin were used to obtain a derived amino acid sequence for a partial cDNA of the isolated protein. The Mytilus protein was 58\% and 77\% identical to C. elegans and
Aplysia twitchin, respectively. An affinity-purified polyclonal antibody made against a peptide in the derived sequence bound to the catch-relaxing protein. Based on these findings, we concluded that the catch-relaxing protein is the Mytilus isoform of the minititin twitchin [63].

\section{Primary Structure and Domain Organization of Mytilus Twitchin}

The amino acid sequence derived from the full length cDNA for twitchin from Mytilus ABRM showed a molecule with a molecular weight of about $526 \mathrm{kDa}$ containing $24 \mathrm{Ig}$ and 15 fibronectin motifs in addition to a single kinase domain [64]. The motif arrangement is shown in Figure 4. A comparison of minititins from Drosophila, crayfish, C. elegans, mosquito and Mytilus shows that all of the known minititins have an $\mathrm{N}$-terminal region consisting of 9 to 12 Ig-like domains. This is followed by a core region containing a variable number of $(\mathrm{Fn})_{2}$ Ig repeats. There are 14 such repeats in Drosophila projectin and only two in Mytilus twitchin. The 


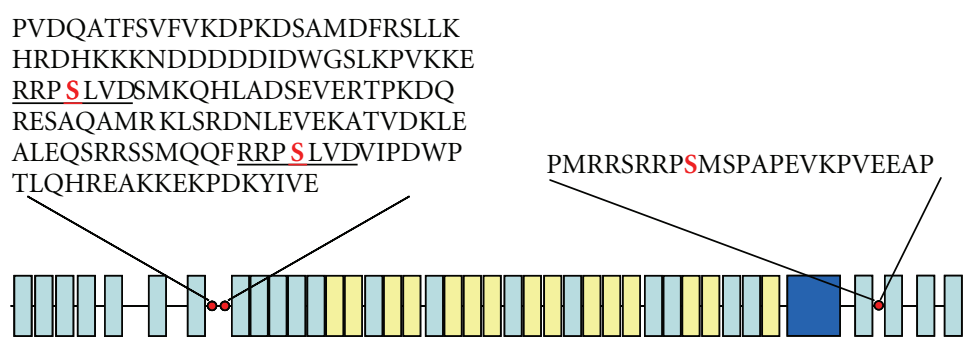

FIGURE 4: The domain structure of Mytilus twitchin. Green, immunoglobulin-like motifs; yellow, fibronectin type III motifs; purple, kinase domain; and red circles, phosphorylation sites. The underlined sequences near the N-terminus share 7 identical amino acids and contain the DX and D1 phosphorylation sites (S). The D2 phosphorylation site (S) is near the N-terminus.

variable number of these structures appears to be the main determinant of the difference in size of the proteins varying from about 530,000 in Mytilus to 1,000,000 in Drosophila. It is not known for what function, if any, the length of the molecule is optimized. The exact number of $(\mathrm{Fn})_{2}$ Ig domains does not appear to be critical, since in C. elegans up to 6 of these domains can be deleted without the animal taking on the twitching phenotype associated with loss of twitchin function $[65,66]$. The next region of the molecule containing the domains $(\mathrm{Fn})_{3} \operatorname{Ig}(\mathrm{Fn})_{2} \operatorname{Ig}(\mathrm{Fn})_{3}\left(\operatorname{Ig}_{2}\right)(\mathrm{Fn})_{2}(\mathrm{Ig})_{2}$ FnKinase is identical in all of the minititins. Lastly, there are either 4 or 5 Ig domains at the $\mathrm{C}$-terminus of the molecule following the kinase domain.

There is considerable variability in the extent to which minititins contain PEVK regions so named for a high incidence of proline $(\mathrm{P})$, glutamic acid $(\mathrm{E})$, valine $(\mathrm{V})$, and lysine (K) residues. Mytilus twitchin includes a 79-residue sequence between Ig domains 6 and 7 (from the $\mathrm{N}$ terminus) in which more than $60 \%$ of the residues are $\mathrm{P}, \mathrm{E}, \mathrm{V}$, or $\mathrm{K}$ [64]. The short length of this segment may not be sufficient to contribute much elasticity to the protein. There is also a short PEVK sequence in mosquito projectin [66]. Drosophila projectin contains a PEVK region (between Ig domains 8 and 9 ) and there appears to be alternative splicing in this region resulting in a host of isoforms of projectin. The PEVK region varies from 100 to 624 amino acids in these different isoforms [67]. The similarity of this region to the PEVK region in titin has led to the expectation that this portion of projectin would, like titin, contribute to the passive elastic properties of the muscle. The same region of crayfish projectin contains a series of twelve 19-amino acid repeats, and each repeat contains a high proportion of glutamic acid, lysine, and valine. Although this region does not show much homology with the PEVK region from Drosophila projectin, it does contribute to the elastic properties of crayfish projectin [68]. Twitchin from C. elegans lacks a PEVK domain as such, but has short regions rich in proline, glutamic/aspartic acid, and serine in the inter Ig domains on each side of the third Ig domain from the N-terminus $[67,69]$.

Mytilus twitchin also contains a DFRXXL motif in the $\mathrm{N}$-terminal portion of the molecule, as do all minititins. The presence of this motif is interesting because studies by Stull and collaborators suggest that it is this motif that is responsible for binding of smooth and nonmuscle myosin light chain kinase (MLCK) to actin [70-75]. The short form of MLCK has its actin-binding site in the N-terminal 142 residues and included in this segment are three DFRXXL motifs that are necessary and sufficient for actin binding. Structural studies show that this N-terminal portion of the short MLCK binds to F-actin at a specific site that is different from, and thus should not interfere with, other actinbinding proteins such as myosin, tropomyosin, caldesmon, and calponin [75]. The presence of the DFRXXL motif in the $\mathrm{N}$-terminal region of all minititins suggests that this portion of the molecules would show a tendency to bind to actin. All of the PEVK-like domains noted above for the minititins are in the inter-Ig domain just N-terminal to the domain containing the DFRXXL motif. Fragments of the PEVK domain of titin from both cardiac and skeletal muscle have been shown to interact with actin and to slow actin sliding over myosin in in vitro motility assays [76]. If the PEVK-like domains bind actin, then the close proximity of the PEVK-like domains and the DFRXXL motif might be additive in their effect on the actin-binding properties of the minititins.

\section{Protein Kinase A Phosphorylation Sites in Mytilus Twitchin}

When twitchin isolated from Mytilus is treated with the catalytic subunit of PKA, the stoichiometry of phosphorylation is about 3 mole phosphate per mole of twitchin. Two phosphorylated peptides have been isolated from tryptic digests of twitchin, and have been identified as D1 and D2. The D2 phosphorylated peptide is located in the linker region between the two Ig domains that are C-terminal to the kinase domain. The D1 sequence is located in the linker region between Ig domains 7 and 8 [64] in the $\mathrm{N}$-terminal portion of the molecule. A third phosphorylated peptide was not successfully sequenced. It was noted, however, that there is a potential phosphorylation site that shares a seven-residue sequence with the D1 site and was located in the same inter-Ig domain as D1. This site was named DX [64]. We have since performed mass spectrometry experiments that show the DX site is phosphorylated when cAMP is added to permeabilized muscles. The mass of the DX peptide in Lys $\mathrm{C}$ digests of twitchin is $1317.5+\mathrm{H}$. In digests of twitchin from muscles in catch, a peptide with this mass was identified, but it was not present in digests of twitchin from muscles 


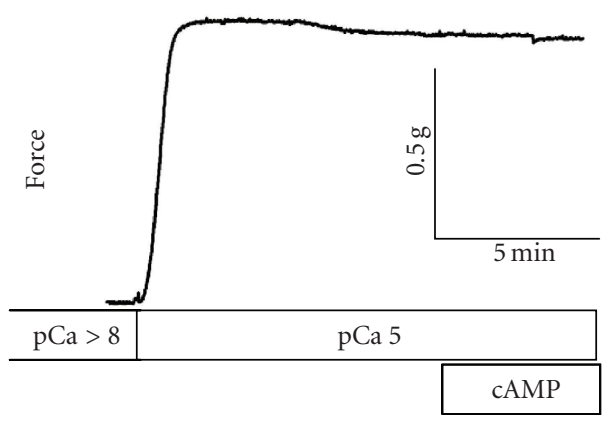

(a)

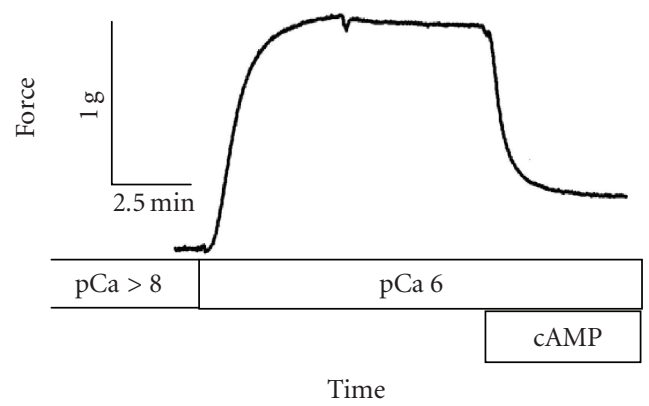

(b)

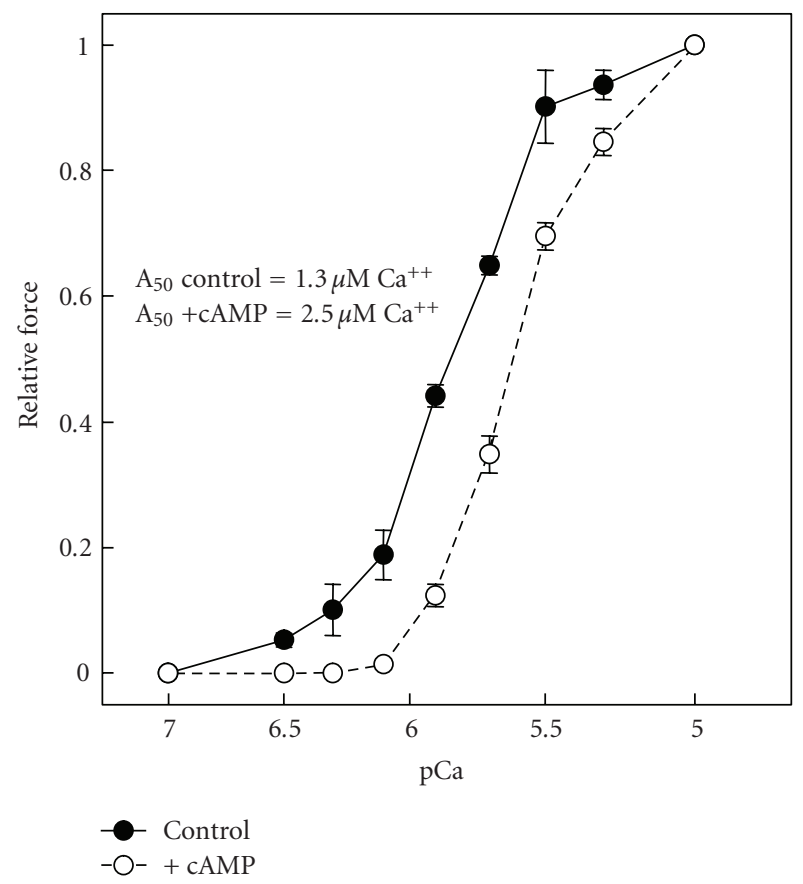

(c)

FIGURE 5: The effect of cAMP on the mechanical responses of permeabilized ABRM following activation at high (pCa 5) (a) and intermediate (pCa 6) (b) concentrations of calcium, and the effect of cAMP on the relationship between isometric force and [Ca ${ }^{2+}$ in permeabilized ABRM (c). In (a) and (b), permeabilized muscles were incubated in relaxing solution, followed by activation with calcium for 10 minutes prior to addition of cAMP $(100 \mu \mathrm{M})$ for 6 minutes. In (c), muscles were treated in a cumulative manner with increasing concentrations of calcium. After a series of steady-state force measurements at a number of incrementing $\left[\mathrm{Ca}^{2+}\right]$ were completed, a transition was made to calcium solutions containing cAMP. Force responses were normalized to the maximum force at pCa5 + cAMP. $T=20 \mathrm{C}$. Reproduced from [63] with permission.

that were subjected to cAMP treatment. Treatment of these digests with Lambda phosphatase caused a reappearance of the $1317.5+\mathrm{H}$ peptide. These experiments suggest that in addition to phosphorylating the D1 and D2 sites, the DX site in twitchin is also phosphorylated by PKA when cAMP is added to permeabilized muscles and catch force is relaxed.

\section{Relationships Among Twitchin D1 and D2 Site Phosphorylation and Relaxation of Catch Force}

Phosphorylation-sensitive antibodies were used to determine how the phosphorylation state of the D1 and D2 sites of twitchin varied under different mechanical conditions [64]. In intact muscles in catch the D2 site was less than $10 \%$ phosphorylated while the D1 site was about $40 \%$ phosphorylated. Addition of serotonin to the muscle relaxed catch force and caused maximum phosphorylation of both sites. The increase in phosphorylation is reversed when the muscle is subsequently put into the catch state. These results are consistent with the idea that phosphorylation of both sites causes relaxation of catch force and that maintenance of catch force requires full dephosphorylation of the D2 site and dephosphorylation of the D1 site in at least half of the twitchin molecules. D1 site phosphorylations from 0 to $50 \%$ appear to have little effect on catch force whereas higher values are associated with relaxation of catch. On the other 
hand, phosphorylation levels in the D2 site exceeding 15\% were associated with relaxation. Interestingly, for both intact and permeabilized muscles, phosphorylation of D2 to more than $15 \%$ only occurred when D1 phosphorylation was $50 \%$ or greater. Therefore, relaxation of catch only occurs when both the D1 and D2 sites are phosphorylated, and the D1 site is more readily phosphorylated than the D2 site. The maintenance of catch force when up to $50 \%$ of twitchin molecules have the D1 site phosphorylated shows that phosphorylation of this site alone does not cause detachment of the catch link. On the other hand, full relaxation of catch force occurs only when the D1 site is fully phosphorylated. Phosphorylation of this site is therefore necessary, but not sufficient to detach the catch link.

Twitchin isoforms have been identified in muscles from Mytilus galloprovincialis [77]. All of the isoforms contain the kinase domain and the D2 phosphorylation site, but they show different sequences in the linker region containing the DX and D1 phosphorylation sites resulting from alternative splicing. In some isoforms a 63 amino acid sequence containing the D1 phosphorylation site is deleted. These isoforms are present only in muscles that do not exhibit catch. In other words, the D1 phosphorylation site is always found in catch muscle twitchin. These data led the authors to conclude that the D1 site of twitchin is "essential to the mechanism of catch" [77].

\section{Catch Force is Present in Submaximally Activated Muscles}

Phosphorylation of twitchin has no effect on force at calcium concentrations required for maximum force production, but decreases force at calcium concentrations that yield submaximum force production [63]. This observation can be summarized as a twitchin-phosphorylation-dependent shift in the force-calcium relationship as shown in Figure 5. At intermediate calcium concentrations, phosphorylation of twitchin causes a decrease in force with little or no associated change in ATPase activity [8]. The "extra" force that is maintained when twitchin is unphosphorylated does not require an "extra" ATPase activity which makes it very unlikely that the extra force is due to normal ATP-driven myosin crossbridge cycling. Rather, the extra force has a very high economy (i.e., very low energy demand) as does catch force maintenance. This suggests that the mechanism that gives rise to catch force maintenance can operate to some extent together with that of cycling myosin crossbridges.

\section{The Structure Responsible for Catch Force Can Adjust During Shortening if the Muscle Is Activated}

One of the hallmarks of the catch state is the absence of force redevelopment following a quick release in length. This has been interpreted to mean that the catch force-maintaining structures do not cycle and redevelop force when muscle length is decreased. The observation that catch force exists during activation at intermediate calcium concentrations

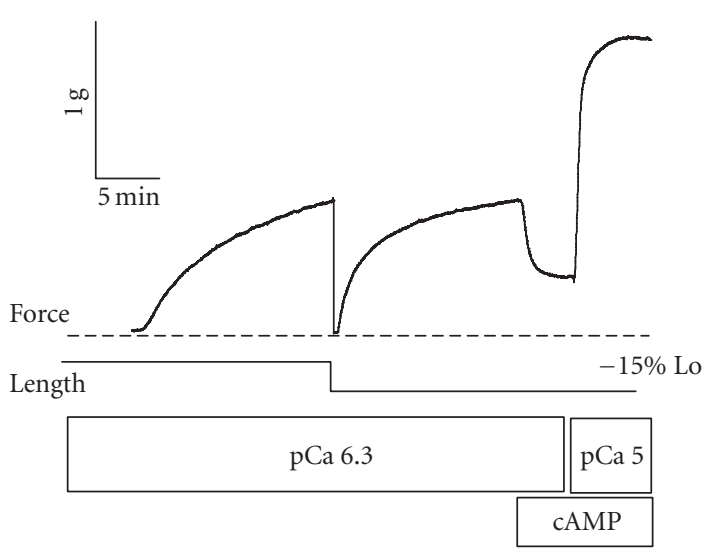

FIGURE 6: Effect of muscle shortening and force redevelopment on cAMP-dependent force production at pCa 6.3 in permeabilized ABRM. After activation in pCa 6.3 for $\sim 15$ minutes, the muscle was subjected to a quick release of $15 \%$ Lo. Force quickly fell to zero and then redeveloped. $100 \mu \mathrm{M}$ cAMP was then added to the bathing medium, and force decreased. The muscle was then maximally activated in pCa5 in the presence of cAMP. $T=20 \mathrm{C}$. Reproduced from [8] with permission.

raised the question as to whether the structures responsible for catch force can cycle when the muscle is activated. If the structures adjust during muscle shortening, then catch force could be redeveloped and subsequently maintained at a new shorter muscle length. This was tested in a muscle activated at submaximal calcium ( $\mathrm{pCa}$ 6.3) and then subjected to various quick releases in the range 10\%-30\% Lo. The large releases that caused force to decrease to zero would be expected to cause a buckling or detachment of all of the force-maintaining structures. Force redeveloped following the release, and, as shown in Figure 6, addition of cAMP and phosphorylation of twitchin still resulted in a decrease in force [8]. This means that some of the redeveloped force was due to structures that are dependent on twitchin phosphorylation. That is, the catch force-maintaining structures present following activation can detach upon shortening and subsequently reattach to maintain newly developed force at the shorter muscle length. Even though the structures responsible for catch force do not require an energy input for force maintenance, they do appear to readjust (cycle) when an activated muscle is allowed to shorten. The fact that redevelopment of catch force following a quick release only occurs in activated muscles suggests that cycling myosin crossbridges may be required for catch force-maintaining structures to readjust and contribute to the redeveloped force.

\section{In Activated Muscles, the Presence of Catch Does Not Affect the Kinetics of Crossbridge Cycling under Isometric Conditions, but Does Alter the Kinetics During Shortening}

The rate of myosin crossbridge cycling was measured as the single turnover of myosin-bound ADP. The basic premise of 
the method is that most myosin has ADP bound at any given time, and that the time course of release of the bound ADP and replacement with new ADP subsequent to ATP binding and splitting gives an accurate measure of kinetics of myosin crossbridge turnover. This method was used to test whether twitchin phosphorylation in activated muscles changed the kinetics of myosin cycling. Under isometric conditions, there is no effect of twitchin phosphorylation on the rate of myosin crossbridge turnover at a calcium concentration that gives submaximal force output, even though force output decreased as a result of the phosphorylation [78]. This supports the view that removal of catch links has no effect on myosin crossbridge turnover under isometric conditions at intermediate calcium concentrations.

In the 1920s, Fenn described experiments that showed that muscles that shortened and performed external work liberated a higher total energy than muscles kept under isometric conditions [79]. This observation means that the rates of the energy producing reactions in muscle change as a function of the mechanical conditions. Many years later, Huxley formulated a model of muscle contraction in which the rate constants for the attachment and/or detachment of myosin and actin depend on the amount of strain on the myosin crossbridge [80]. More recent experiments suggest that the rate constant for ADP release from actin-attached myosin is one of the main strain-dependent steps in the myosin crossbridge cycle [81]. High force conditions (associated with isometric conditions) would tend to minimize the movement of the myosin crossbridge and keep ADP release slow, whereas low-force conditions following a decrease in muscle length would lower the strain on the crossbridge and increase both the rate of ADP release as well as the overall rate of myosin crossbridge cycling.

The strain dependence of myosin crossbridge turnover in catch muscle was determined by comparing myosin-bound ADP turnover under isometric and shortening conditions [78]. When a muscle is maintaining catch force at very low (basal) calcium concentrations, no strain dependence following a quick release was detectable. On the other hand, at calcium concentrations that result in near-maximal activation (no catch component of force output), there is an approximately three-fold increase in the rate constant for myosin-bound ADP turnover when the crossbridge is unstrained by a quick release compared to isometric conditions. The use of similar methods showed about a five-fold increase in myosin-bound ADP turnover following a quick release in mammalian smooth muscle [82]. In addition, Khromov et al. [83], using different methods, found about a two-fold increase in the rate constant for ADP release from myosin with a decrease in strain in mammalian smooth muscle. So, the fully activated catch muscle shows a similar increase in myosin crossbridge turnover as do other smooth muscles.

Surprisingly, at intermediate calcium concentrations where the muscle is only partially activated, there was no significant increase in the rate of turnover of myosin following a quick release when twitchin was unphosphorylated. In contrast, when twitchin is phosphorylated and the catch component of force output is thereby removed, there is an increase in myosin turnover associated with shortening. Clearly, the presence of the catch force-maintaining structure alters the response of myosin to a decrease in muscle length. It is possible that the catch link prevents the immediate motion of the myosin head following the quick release. This could occur if the link tightly connects thick and thin filaments and prevents or slows the relative motion of the filaments following release. A slowing in the relative motion of the filaments by such a linkage would delay much of the expected increased turnover of myosin-bound ADP following a quick release. Since the single turnover protocol only measures the kinetics of the first turnover of each myosin-bound ADP, the ADP turnover on myosin for the first crossbridges that cycle following a quick release would be similar to isometric conditions, whereas the crossbridges that cycle later could have a higher rate reflecting the progressive detachment of the catch link as the shortening progresses.

\section{Is Catch Force Maintenance Due to the Unique Kinetics of Myosin Crossbridges or is it Due to the Presence of Some Other Protein Linking Thick and Thin Filaments When Myosin Crossbridges Detach?}

Several studies have attempted to determine which of the two prevailing theories of catch, described above, was correct. The fact that catch force-maintaining structures appear able to slowly detach and reattach during and following muscle shortening (as does myosin) led us to initially favor the view that myosin-actin linkages are responsible for the maintenance of catch force $[8,84]$. However, as discussed below, the preponderance of current evidence favors the idea that there is a link between thick and thin filaments other than the myosin crossbridge that mediates catch. Some of the unique mechanical aspects of catch that support this view are summarized in the following findings.

13.1. Catch Force Remains Following Detachment of Myosin Rigor Crossbridges by Addition of MgATP. The relationship between the catch link and myosin crossbridge detachment from actin was studied by determining how rigor force relaxes following addition of MgATP [84]. Addition of MgATP to permeabilized muscles in high-force rigor in the absence of calcium resulted in a rapid loss of a small component of force followed by a very slow rate of relaxation that is characteristic of catch (Figure 7). Subsequent addition of cAMP causes a relaxation of the remaining force. If twitchin is thiophosphorylated before the addition of ATP, no catch component remains. These results show that catch force persists following detachment of rigor myosin crossbridges as long as twitchin is unphosphorylated. A similar dependence of force relaxation from rigor on twitchin phosphorylation is seen with AMP-PNP and ATP $\gamma$ S (nonhydrolysable analogs of ATP). This suggests that the catch force persistence is not due to crossbridges that detach and then go through cooperative reattachment to actin [85] since there would be no hydrolysis of nucleotide to ADP that necessary as 


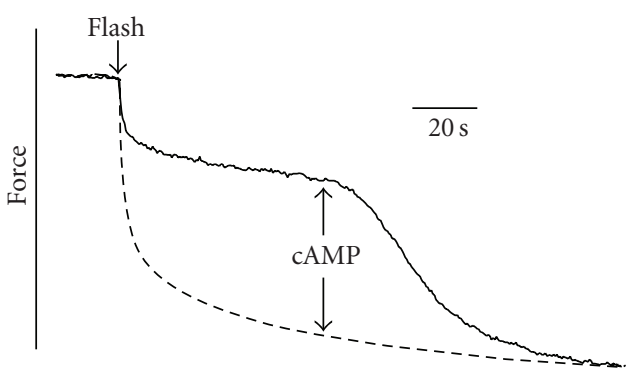

FIGURE 7: Force responses following release of ATP from caged ATP in permeabilized ABRM. Muscles were put into high-force rigor by activation in $\mathrm{pCa} 5$ and then transferred to a pCa5rigor solution containing apyrase $(0.2 \mathrm{mg} / \mathrm{mL})$. Calcium was then lowered to $\mathrm{pCa}>8$. Caged ATP $(4 \mathrm{mM})$ was added $4 \mathrm{~min}$ before the flash and the muscle was transferred to a solution containing $1 \mathrm{mM}$ MgATP $\sim 4 \mathrm{~s}$ following the flash. cAMP was added at the time shown. Solid line, twitchin not phosphorylated; dashed lines, twitchin thiophosphorylated. $T=20$ C. Reproduced from [84] with permission.

a prelude to attachment. Under rigor conditions, where there is little if any ADP bound to myosin, the burst of ADP formation following addition of ATP (measured from ${ }^{3} \mathrm{H}$-ADP formation following photolysis of caged ${ }^{3} \mathrm{H}$-ATP) equals the myosin head concentration and is not dependent on the state of twitchin phosphorylation. This means that all of the myosin binds ATP and that catch force is not due to rigor crossbridges that persist following addition of ATP. It also argues against the possibility that there is a significant fraction of myosin having ADP bound in the rigor muscle that could possibly be responsible for continued force maintenance after addition of ATP. These results strongly suggest that if catch force is maintained by myosin crossbridges, then ATP binding to the rigor catch crossbridge does not lead to detachment of the catch crossbridge even though ATP is rapidly hydrolyzed. The experiments also show that if myosin crossbridges are responsible for catch force maintenance, then they have ADP bound. Of course, such experiments are also consistent with a mechanism in which catch force is maintained by an independent link between thick and thin filaments that is not mediated by the myosin crossbridge.

13.2. Relaxation of Catch Force by Phosphorylation of Twitchin Is Not Associated with a Measurable Turnover of MyosinBound ADP. If myosin crossbridges with ADP bound interact with actin to maintain catch force, then relaxation of catch force by twitchin phosphorylation should bring the crossbridge back to the resting state by completion of the myosin crossbridge cycle. This would involve the release of ADP followed by ATP binding, crossbridge detachment, and subsequent ATP splitting with ADP and Pi remaining bound to the myosin. The results of single turnover experiments showed that a major fraction of myosin does not turn over ADP during the detachment of catch force-maintaining links [78]. It is therefore unlikely that relaxation of catch involves the detachment of myosin from actin through steps normally occurring during completion of the myosin crossbridge cycle. Although such experiments do not rule out the involvement of an actin-myosin crossbridge attachment in catch force maintenance, they make it less likely since detachment of myosin from actin during relaxation of catch would have to be different from that exhibited by every other myosin II studied.

\section{Is the Catch Force-Maintaining Structure a Myosin Crossbridge Whose Detachment Is Prevented by Unbinding of Calcium?}

A scheme that would include myosin in catch force maintenance is based on a modification of the original "latchbridge" model for the regulation of force output in mammalian smooth muscle [86]. In this model, phosphorylation of the regulatory light chain of myosin results in myosin crossbridge cycling with force development and maintenance. Subsequent dephosphorylation of the myosin light chain while myosin is attached to actin and generating high force results in a large decrease in the rate constant for detachment of the unphosphorylated crossbridge. In this way, high force would be maintained by the dephosphorylated myosin crossbridge with little associated myosin ATPase activity, and with little myosin light chain phosphorylation. It is now known that there are other mechanisms that play a role in the maintenance of force by myosin with unphosphorylated light chains. These include cooperative activation of unphosphorylated crossbridges by phosphorylated crossbridges [85, 87-91] and possibly tight binding of ADP to myosin giving rise to a slow detachment rate from actin associated with accumulation of ADP [92]. Even with these other mechanisms the idea that dephosphorylation of attached myosin leads to slowed detachment from thin filaments and results in force maintenance is an attractive mechanism.

Application of the latch model to catch muscle requires two major modifications. Since invertebrate catch smooth muscle is regulated directly by calcium binding, the binding of calcium to and release from myosin would replace the phosphorylation and dephosphorylation of the myosin light chain. The other modification is that the detachment rate constant of the catch crossbridge would presumably be regulated by the state of phosphorylation of twitchin. In such a myosin crossbridge model of catch, calcium binding to myosin initiates myosin interaction with actin, and the myosin crossbridge would go through normal cycles involving attachment to actin, development of force, and subsequent detachment. But unbinding of calcium while the myosin is in the actin-attached high force state would lead to a catch crossbridge whose detachment rate constant would be very slow. Phosphorylation of twitchin would increase the detachment rate constant for the calcium freecatch crossbridge, and relax catch force. Myosin could also detach from actin by rebinding calcium and re-entering the normal crossbridge cycle. Such a general model of catch involving the myosin crossbridge could account for many of the mechanical responses in intact [93] and permeabilized catch muscle [8]. 


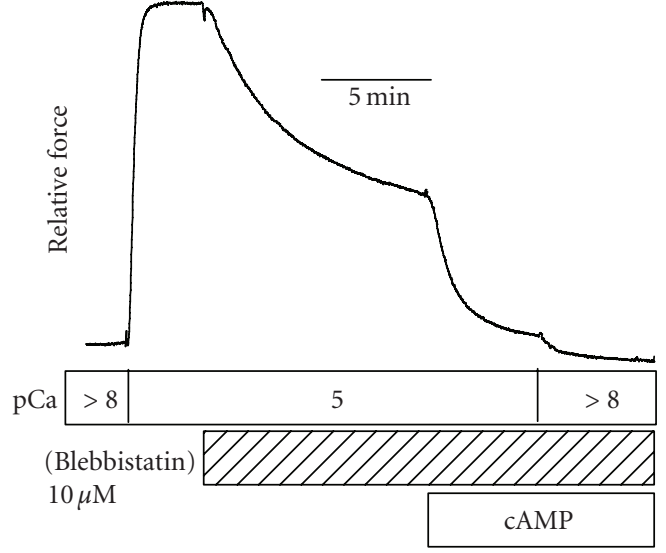

Figure 8: Effect of blebbistatin on force output and on the sensitivity of force to twitchin phosphorylation in permeabilized ABRM. Note that the addition of cAMP after blebbistatin treatment decreased force in $\mathrm{pCa} 5$ to nearly that present in relaxing solution ( $\mathrm{pCa}>8$ ). $T=20 \mathrm{C}$. Reproduced from [98] with permission.

\section{How Is Catch Force Modified by Agents that Inhibit the Transition from Low Force to High Force in Myosin?}

The amount of catch force that exists under different mechanical conditions in permeabilized muscles can be quantified by determination of the amount of force that is relaxed upon the addition of cAMP and associated phosphorylation of twitchin. This allows the assessment of how catch force depends on factors that modify myosin interaction with actin and provides a powerful tool in probing the mechanism responsible for catch force maintenance. Such experiments provide a direct means of determining whether the catch force-maintaining structure is a myosin crossbridge that is attached to actin, whose detachment is prevented by the unbinding of calcium from myosin.

Blebbistatin inhibits the actin-activated ATPase activity of myosin II $[94,95]$ by preventing phosphate release from the myosin head, and thus trapping myosin in the ADP and phosphate-bound state with low actin affinity [96]. Blebbistatin acts by binding to the $50 \mathrm{kDa}$ cleft of myosin near the $\gamma$-phosphate-binding site [97], thereby keeping the myosin crossbridge in the weak actin-binding state. Blebbistatin is an effective inhibitor of myosin in catch muscle since it totally inhibits the increase in ATPase activity when calcium is increased from pCa $>8$ to pCa 5 [98]. The effect of blebbistatin on force output in catch muscle at pCa 5 is shown in Figure 8. Under these conditions, blebbistatin causes a partial relaxation of force that can be fully relaxed by the addition of cAMP. As expected, the cAMP results in phosphorylation of twitchin. This twitchin phosphorylation-dependent decrease in force was unexpected because previous experiments at high calcium had shown that there is no such effect in the absence of blebbistatin. Since the residual force during treatment with blebbistatin is not associated with a measurable myosin ATPase activity and is relaxed by twitchin phosphorylation, it

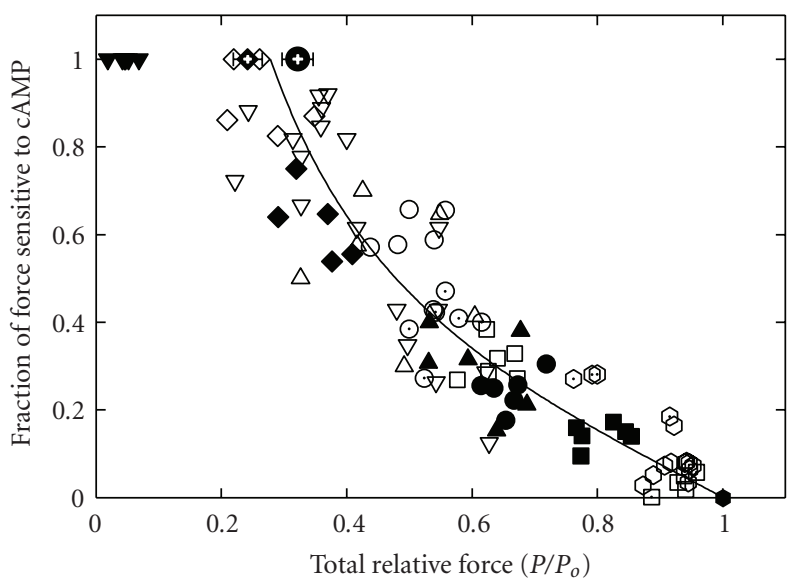

Figure 9: Relationship between the fractional decrease in force caused by phosphorylation of twitchin and the total force $\left(P / P_{o}\right)$ before twitchin phosphorylation in permeabilized ABRM. Data for individual muscles are shown as the following: pCa 4.5, $+P_{i}(\bullet)$; pCa 5, control $(\bullet), P_{i} 5 \mathrm{mM}(\diamond), P_{i} 25 \mathrm{mM}(\odot)$, blebbistatin $1.25-$ $25 \mu \mathrm{M}(\nabla)$; pCa 5.7, control (ם), $P_{i} 5 \mathrm{mM}(\square)$; pCa 6, control $(\bullet$ $\mathbf{\Delta}), P_{i} 5 \mathrm{mM}(\bigcirc), \mathrm{BDM} 10 \mathrm{mM}(\triangle)$, TFP $200 \mu \mathrm{M}(\odot)$; pCa 6.3 $(\checkmark), P_{i} 5 \mathrm{mM}(\square)$; pCa $7,(\boldsymbol{\nabla})$. Mean $\pm \mathrm{SE}(N=16)$ for catch force remaining 10 minutes $(\bullet), 20$ minutes $(\bullet)$ after switch from pCa 6 to $\mathrm{pCa}>8$. The line is a least squares quadratic fit to the data. $T=$ 20 C. Reproduced from [98] with permission.

is catch force. Therefore, catch force can be maintained under high calcium conditions in the presence of blebbistatin. Such results are not consistent with the idea that catch force is maintained by a high force actin-attached myosin crossbridge whose detachment is prevented by the unbinding of calcium from myosin. Calcium is still present under these conditions and calcium-bound high force myosin would be expected to have a high rate of detachment from actin. This detachment, in addition to the blebbistatin-mediated inhibition of the transition of the myosin crossbridges into the high-force state would result in all of the myosin being in the weakly bound low-force state and, thereby, unable to maintain catch force in this model.

Other inhibitors of the low-to-high force transition of the myosin crossbridge including inorganic phosphate, butanedione monoxime, and trifluoperazine caused a decrease in total force and an increase in the catch force, measured as relaxation on addition of cAMP and phosphorylation of twitchin [98]. A decrease in force for any reason (other than twitchin phosphorylation) may be inherently associated with an increase in catch force. It may be that the total force itself determines the amount of catch force present, rather than other factors such as calcium concentration. For example, the amount of catch force may be the same at a force of $50 \%$ Po whether that force was generated in a muscle activated with submaximal calcium concentrations or in a muscle that is maximally activated in high calcium in the presence of an inhibitor of the low-to-high force transition of myosin. Figure 9 shows the relationship between the fraction of force that is catch and the total force output of the muscle. At high forces there is little catch force, and at low forces the 


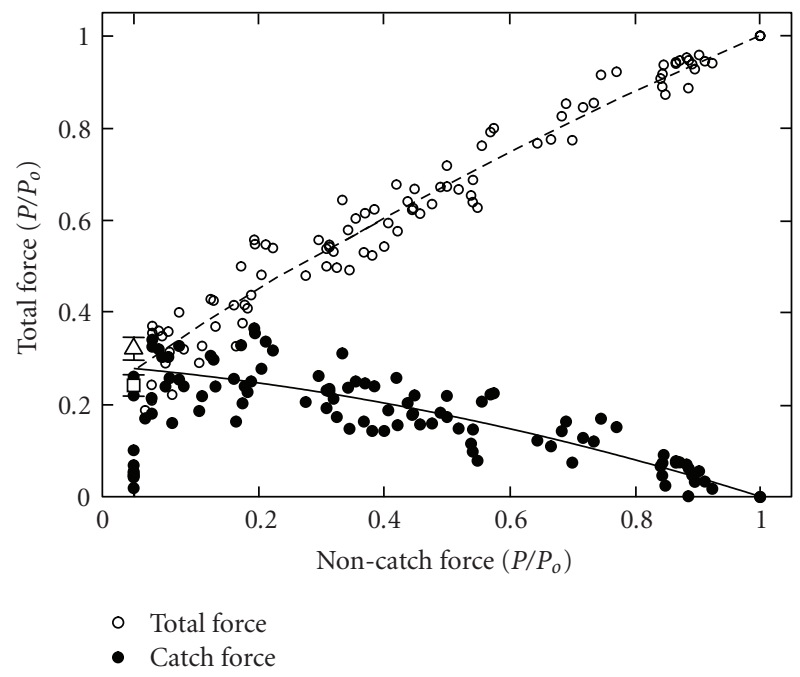

Figure 10: Dependence of total force and catch force on noncatch force under various conditions in permeabilized ABRM. The data are derived from the same experiments shown in Figure 9. Noncatch force is that remaining after addition of cAMP, while catch force ( solid line) is the change in force resulting from addition of cAMP. Total force $(\bigcirc$, dashed line $)$ is the force before cAMP addition. Also shown are the mean \pm SE for catch force remaining 10 minutes $(\triangle)$ and 20 minutes $(\square)$ after switch from pCa 6 to $\mathrm{pCa}>8$. The lines are least squares quadratic fits to the data. Reproduced from [98] with permission.

fraction of total force that is catch increases. At a given total force, the amount of catch force is not affected by the calcium concentration or presence of the above inhibitors.

Total force output can be apportioned into catch force and noncatch force which is presumably due to cycling myosin crossbridges using the data shown in Figure 9. Since twitchin phosphorylation does not change the muscle ATPase activity or the turnover of myosin-bound ADP, crossbridge cycling and its associated noncatch force are not modified by twitchin phosphorylation. Figure 10 shows the dependence of total and catch force on noncatch force. When the force output from myosin crossbridge cycling is high, catch force is low. As the force from myosin crossbridge cycling decreases, catch force increases almost linearly to about $30 \%$ of total force.

\section{Is the Catch Structure a Molecule Other than Myosin That Can Bind Both Thick and Thin Filaments and Which Can Be Displaced from the Thin Filament by High-Force Myosin Binding to Actin?}

The data suggest that myosin in the high-force state is associated with detachment of catch links whereas myosin in the low-force state promotes formation of catch links. It is possible that myosin interacts with the catch link in such a way that when myosin is attached to actin in the high force state it displaces the catch link from actin, preventing any catch force maintenance. When myosin detaches from actin and enters the low force state, the catch link has access to actin-binding sites and the force-maintaining catch link can reform. Phosphorylation of twitchin will also detach the catch link regardless of the state of activation of the muscle. The binding of the catch link to the thin filament may be regulated by structural changes in the thin filament caused by interactions of the myosin crossbridges.

A mechanism that includes a myosin crossbridgemediated control of the binding of an independent catch link to the thin filament could be the basis of several of the mechanical and biophysical results obtained. Some examples are the following. (1) Catch force maintenance with no associated ATPase and no effect of twitchin phosphorylation on myosin crossbridge kinetics under isometric conditions. The catch link itself has no ATPase activity and any force that is maintained results from myosin crossbridge cycling or resistance to lengthening of the muscle. Also, the detachment of the catch link would not be expected to affect myosin crossbridge turnover so there would be no change in myosin ATPase or myosin-bound ADP turnover under isometric conditions as a result of twitchin phosphorylation. (2) Catch force inhibits the strain-dependent increase in myosin-bound $A D P$ turnover during shortening at intermediate calcium concentrations, but not under maximally activated conditions. The catch linkage which provides a connection between thick and thin filaments would be expected to partially interfere with the relative motion of the filaments following release of the muscle. This would slow the initial cycling of myosin. Detachment of the linkage by either myosin binding as described above or by the relative translation of filaments resulting from some myosin cycles would subsequently allow robust shortening of the muscle. There would be no such interference with relative translation of the filaments when the muscle is fully activated since no catch link is present before the shortening. The expected increase in rate constant for single turnover of myosin-bound ADP occurs under this condition. (3) Catch force maintenance coexists with myosin crossbridge cycling. Catch force maintenance would be present under all conditions except when the maximum number of myosin crossbridges is attached to actin. When a myosin crossbridge detaches from actin, the catch linkage forms between thick and thin filaments. If the linkage forms after the crossbridge detaches from the thin filament, then the linkage cannot maintain the force generated from that crossbridge. But the catch link would maintain some of the force from myosin crossbridges that are attached when the catch linkage forms, but which detach during the time that the catch link is still attached. In this way there is, on average, an extra force output resulting from such catch linkages. In effect, the catch linkage extends the duty cycle of myosin crossbridges that are generating force when the linkage is formed and which detach before the catch link. (4) Redevelopment of catch force maintenance following muscle shortening at intermediate calcium concentrations. The catch link would cycle during shortening because myosin would still interact with actin and displace the link which would re-form when the myosin crossbridge detaches. Both catch and noncatch force would redevelop at the new muscle length. (5) Catch force remains following detachment of rigor 
myosin crossbridges by addition of MgATP. If myosin in the high-force actin-attached state displaces the catch link from the thin filament, then one would expect that when the muscle is in high-force rigor, catch links would be detached. But when the rigor myosin crossbridges bind ATP and detach from actin, the catch link would bind to the thin filament. Since not all myosin crossbridges would detach at the same instant, some catch linkages could form before all rigor myosin crossbridges detach. In this case, these linkages would maintain some of the force from such late detaching crossbridges even after they have bound ATP and detached. There would, therefore, be catch force maintenance when ATP is added to a muscle in high-force rigor.

\section{The Twitchin Molecule is a Likely Candidate for the Catch Link Between Thick and Thin Filaments}

Since the phosphorylation state of twitchin controls catch force maintenance it is an obvious candidate for the catch link. In vitro motility studies on isolated proteins including thick filaments formed from only purified myosin, F-actin and twitchin have shown mechanical results consistent with the catch state [19]. When added to permeabilized human skeletal muscle fibers, twitchin isolated from molluscan catch muscle causes a "catch-like stiffness" which decreases when twitchin is phosphorylated [99]. This strongly suggests that twitchin is sufficient to mediate catch behavior between thick and thin filaments.

Muscles that do not exhibit catch may also contain twitchin, but their twitchin content is considerably less than that of catch muscles. For example, there is 3-fold less twitchin per myosin heavy chain in the scallop obliquely striated adductor muscle than in the smooth adductor muscle that shows catch. The twitchin from noncatch muscles also shows the ability to confer catch-like properties on isolated proteins [100]. This suggests that the twitchinmediated interaction between thick and thin filaments may occur in other than catch muscle and that such interactions may modulate relaxation rate [100]. The amount of twitchin, and perhaps the specific isoform present (see [77]) may determine the extent to which classical catch properties are exhibited by a particular type of muscle.

\section{Biochemical Evidence that Twitchin is the Catch Link}

18.1. Twitchin Binds to the Thick Filament. In molluscan catch muscle, an antibody against twitchin binds along the entire length of isolated thick filaments as well as thick filaments in tissues as observed in immunoelectron microscopy [61]. This is also shown in greater structural detail in Figure 11, in which the kinase domain of twitchin was immunogold labeled in isolated thick filaments from the ABRM of Mytilus edulis (Siegman and Butler, unpublished). Comparison of the Coomassie Blue staining of twitchin and myosin heavy chain in gels containing whole protein extracts from intact ABRM of Mytilus edulis show that twitchin is

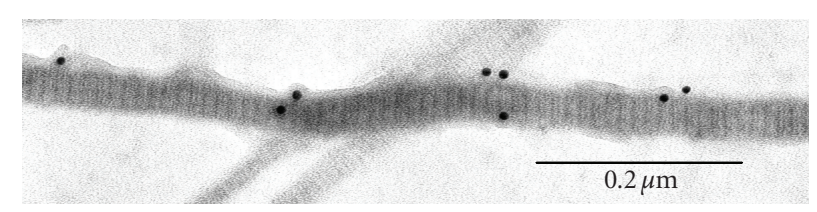

FIGURE 11: Thick filament from ABRM with immunogold labeling of the kinase domain of twitchin. Gold particles are $10 \mathrm{~nm}$ in diameter. Filaments were stained with $2 \%$ uranyl acetate. Striated appearance of filaments has a $\sim 14 \mathrm{~nm}$ repeat and there is about $200 \mathrm{~nm}$ between clusters of gold particles.

$9 \%$ of myosin heavy chain. Given the difference in molecular weights, there is one twitchin for every 14-double-headed myosins [58]. A similar ratio of twitchin to myosin heavy chain was found in whole muscle samples as in isolated thick filaments (Butler and Siegman, unpublished observations). These data show that most of the twitchin is bound to the thick filament tightly enough to remain attached during the thick filament isolation procedure [101]. In vitro binding of twitchin has been shown to myosin, paramyosin, and catchin, all of which are components of the thick filaments [102]. Although twitchin binds to both the rod and heavy meromyosin portions of myosin, binding to the latter was stronger. Phosphorylation of twitchin only slightly decreased the extent of twitchin binding to myosin [102].

18.2. Twitchin Binds to the Thin Filament. Turbidity, viscosity and cosedimentation experiments show that twitchin interacts with F-actin [103]. The interaction was not dependent on calcium concentration, but was highly regulated by the phosphorylation state of twitchin. Twitchin that was treated with the catalytic subunit of protein kinase A did not interact with actin. It is interesting that under some conditions, twitchin appeared to crosslink thin filaments such that co-sedimentation of twitchin and F-actin mixtures occurred at centrifugal forces at which the individual proteins do not pellet. This suggests that there are at least two actin-binding regions in the molecule.

18.3. The C-Terminal Portion of Twitchin Containing the D2 Phosphorylation Site and Adjacent Ig Domains Forms a Trimeric Complex with Myosin and Actin. The D2 regulatory phosphorylation site in the C-terminal portion of twitchin is located in a linker region between the Ig domains that are immediately adjacent to the kinase domain. A recombinant protein containing these Ig domains and the linker region (IGD2IG) has been shown to bind to Factin, myosin, and paramyosin [104]. Thiophosphorylation of the D2 site (which mimics phosphorylation) causes a decrease in binding of IGD2IG to all of these proteins. Cosedimentation of a trimeric complex of F-actin, myosin, and IGD2IG occurred only when IGD2IG was unphosphorylated. The IGD2IG-binding site on actin was identified by making enzymatic digests of actin and determining which of the peptides bound to the unphosphorylated twitchin fragment. Only one peptide with a sequence of LVCDNGS bound to IGD2IG [104]. This sequence is located near the 
sequence of actin that interacts with the loop 2 region of myosin, and a synthetic peptide containing the sequence was found to compete with actin for binding of IGD2IG. Thiophosphorylation of the D2 site prevents the binding of the actin peptide [104]. Additional studies have shown that a peptide derived from the myosin loop 2 sequence (CAQNKEAETTGTHKKRKSSA) binds to IGD2IG and also interferes with formation of the trimeric complex among F-actin, myosin, and IGD2IG [105]. These results suggest IGD2IG links myosin and actin by binding to the loop 2 portion of myosin and to the region of actin where the loop 2 region of myosin binds [105]. Taken together, these results support the idea that the D2 region of twitchin is part of the mechanical link tethering thick and thin filaments and is at least partially responsible for catch force maintenance. The fact that the IGD2IG binds to the loop 2 region of myosin and its corresponding binding site on actin suggests that the binding of myosin to actin during crossbridge cycling might prevent IGD2IG binding and remove the twitchin- mediated tether between thick and thin filaments. This possibility is supported by the finding that addition of calcium and resulting myosin crossbridge cycling decreased the co-sedimentation of the trimeric complex of myosin, actin, and IGD2IG [105].

18.4. The N-Terminal Portion of Twitchin Containing the DX and D1 Phosphorylation Sites Binds Both Thick and Thin Filaments. The DX and D1 regulatory phosphorylation sites are located in the linker region between the 7 th and 8 th IG domains from the N-terminus of twitchin. This linker region also contains a DFRXXL actin-binding motif. Our experiments involving a recombinant protein containing this linker region and adjacent IG domains (IGDXD1IG) show phosphorylation-dependent co-sedimentation of the protein with both native thick and thin filaments from the ABRM of Mytilus edulis. Similar phosphorylation-dependent cosedimentation with thick and thin filaments occurs with a recombinant protein (DXD1) consisting of only the linker region containing the two phosphorylation sites. The binding of DXD1 to native thin filaments shows a $\mathrm{Kd}$ of approximately $16 \mu \mathrm{M}$ and a maximum binding of 1 mole per mole of actin. Phosphorylation of DXD1 increases the $\mathrm{Kd}$ by about 6 -fold if it is assumed that maximum binding is 1 mole per mole of actin. Unphosphorylated IGDXD1IG shows somewhat tighter binding to actin ( $\mathrm{Kd}<5 \mu \mathrm{M})$. Both of these unphosphorylated proteins increase force output in permeabilized ABRM under conditions of submaximal activation, and this effect is not present when the proteins are phosphorylated. This suggests that both IGDXD1IG and DXD1 are sufficient for tethering thick and thin filaments and for adding a catch force component to total force output from the muscle.

There appear to be at least two thin filament interaction sites in the DXD1 linker region. Both a 52-residue peptide surrounding the DX site (which also contains the DFRXXL actin-binding motif) and a 47-residue peptide surrounding the D1 site show co-sedimentation with native thin filaments. Addition of these peptides to permeabilized muscles causes an increased rate of relaxation of catch force. This result is consistent with these peptides displacing native twitchin from actin during catch and confirms the central role that the DXD1 region plays in the mechanism of catch. Interestingly, in muscles in which twitchin has been thiophosphorylated and all force is due to cycling myosin crossbridges, the unphosphorylated DX peptide inhibits force output. When the DX peptide is phosphorylated, the extent of inhibition is decreased. These findings suggest that the unphosphorylated DX region of twitchin can interact with actin to either interfere with myosin binding to actin or to prevent the transition of myosin crossbridge from the low-force to highforce state. The inhibition of force by the DX peptide suggests that this region of twitchin may compete with myosin for binding to actin and, as such, may be responsible for the proposed displacement of twitchin from actin and associated loss in catch force when myosin crossbridges interact with actin.

\section{A Belt and Suspenders Model of Catch}

The data are consistent with twitchin being a tether between thick and thin filaments that is responsible for catch force maintenance. Both the $\mathrm{N}$ - and $\mathrm{C}$-terminal portions of twitchin contain phosphorylation sites that regulate catch, and both of these regions have properties consistent with twitchin being an independent tether between thick and thin filaments. A cartoon of two such tethers in a twitchin molecule is shown in Figure 12. The redundancy provided by two force-bearing tethers in each twitchin molecule would provide an especially secure "belt and suspenders" connection between thick and thin filaments and would promote long-term force maintenance and resistance to stretch during catch.

A model for catch that is a modification of one we described earlier [98] is shown in Figure 13. The myosintwitchin-actin interactions described apply independently to both the $\mathrm{N}$ - and C-terminal regions of twitchin as shown in the "belt and suspenders" cartoon. In the relaxed state, (a) the myosin crossbridge is in the low-force state and detached from the thin filament. The DX, D1, and D2 regulatory sites in twitchin are phosphorylated, and twitchin is detached from the thin filament. When the muscle is activated there is an increase in $\left[\mathrm{Ca}^{+2}\right]$ and the crossbridge binds to the thin filament in the low-force state (b). Twitchin is displaced from the thin filament as a result of crossbridge binding to the thin filament. Twitchin is also dephosphorylated by activation of the phosphatase calcineurin. The crossbridge then makes the transition to the high-force state (c) resulting in force generation. Twitchin is still detached from the thin filament because of the myosin interaction with the thin filament. When the calcium concentration decreases, myosin detaches from the thin filament and the unphosphorylated twitchin is attached to both the thick and thin filaments (d). This is the catch state. The asynchronous activity of crossbridge cycling allows some crossbridges to detach from actin with formation of the trimeric catch complex while some other crossbridges are still attached and maintaining 


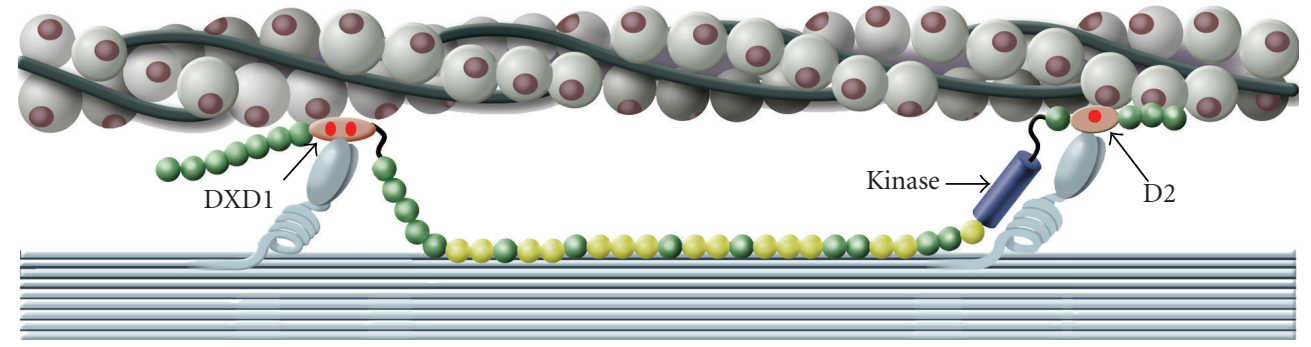

Figure 12: A belt and suspenders model of twitchin interaction with the thick and thin filaments in catch. The redundancy provided by two force-bearing tethers in each twitchin molecule is shown. The $\mathrm{N}$ - and $\mathrm{C}$-terminal portions of twitchin that contain regulatory phosphorylation sites are shown to be independent tethers. The N-terminal tether shows the region surrounding the DX and D1 phosphorylation sites interacting with the thin filament, and the C-terminal tether shows the region surrounding the D2 phosphorylation site interacting with the thin filament. The evidence for the DXD1 region binding to the thin filament comes from this work and the evidence for D2 and adjacent Ig domains comes from [104, 105].

force. The tether formed when the first crossbridges detach will then maintain some of the force of those crossbridges that detach later. In this way force maintenance results from catch linkages when the calcium concentration is decreased. The catch linkage essentially extends the duty cycle of myosin crossbridges that are generating force when the linkage is formed and which detach while the catch link is maintained. Phosphorylation of the regulatory sites in twitchin at resting $\left[\mathrm{Ca}^{+2}\right]$ results in detachment of the tether from the thin filament (and possibly the thick filament), relaxation of catch force, and the loss of the tether-mediated resistance to stretch (Figure 13(a)).

\section{Similarities between Twitchin and Myosin Binding Protein-C (MyBP-C)}

We have previously noted that there are similarities in the domain organization of twitchin around the D1 phosphorylation site with that of cardiac MyBP-C [64]. Although MyBP-C has obviously been known to bind to myosin, there is recent evidence that several regions near the $\mathrm{N}$-terminus of cardiac MyBP-C bind to filamentous actin [106]. One of the actin binding regions includes the cardiac specific regulatory region between domains $\mathrm{C} 1$ and $\mathrm{C} 2$, and phosphorylation of MyBP-C at the cardiac specific sites reduces the interaction with actin [106]. This same region includes a DFRXXLlike actin binding motif (DLRGML) that is similar to that present in twitchin. When unphosphorylated, this regulatory region of cardiac MyBP-C binds to the myosin S2 region [107], but not when phosphorylated [108]. The similarities in actin and myosin binding properties of the DXD1 region of twitchin and the N-terminal portion of MyBP-C as well as their phosphorylation dependence suggest that they may share similar functions. The idea that MyBP-C may be a phosphorylation-dependent tether between thick and thin filaments may seem unusual, given that cardiac muscle goes through rapid and frequent contraction and relaxation cycles. However, if the tethering of the thick and thin filaments via MyBP-C is prevented by myosin crossbridge interaction with the thin filament as suggested for twitchin, then the main effect of such a MyBP-C tether may be to act as a regulated viscous element during relaxation when crossbridge interaction with actin wanes. For example, the rate of relaxation of force seems to be modulated by the phosphorylation of twitchin in Aplysia [62], and the same may be the case for MyBP-C in the heart. Of course, the multiple thick and thin filament tethers present in twitchin from catch muscle would be expected to give a much tighter connection between the contractile filaments and provide a much more prolonged force maintenance following inactivation of catch muscle than would a MyBP-C tether in cardiac muscle.

\section{Summary}

(1) Catch is a mechanical state occurring in certain invertebrate smooth muscles initiated by cholinergic nerve stimulation, and characterized by highforce maintenance and resistance to stretch during extremely slow relaxation. Catch occurs following a transient increase in intracellular calcium and its return to near-basal concentrations. During catch, crossbridge cycling rate is extremely slow as is the rate of ATP utilization.

(2) The release of catch, or rapid relaxation, occurs in vivo upon stimulation of serotonergic nerves, which cause an increase in cAMP and activation of protein kinase A. The primary target of protein kinase A activation is the phosphorylation of the minititin, twitchin. Subsequent activation of the muscle causes an increase in the activity of a calcium-dependent phosphatase, presumably calcineurin, which dephosphorylates twitchin and allows catch force maintenance when intracellular calcium decreases. The simultaneous stimulation of cholinergic and serotonergic nerves results in a phasic contraction.

(3) Twitchin from Mytilus ABRM is a $\sim 526 \mathrm{kDa}$ protein that is associated with the A band of nematode striated and molluscan smooth muscles, specifically with the thick filament. It bears similarity to minititins from other invertebrates with variations in the number of specific domains. 


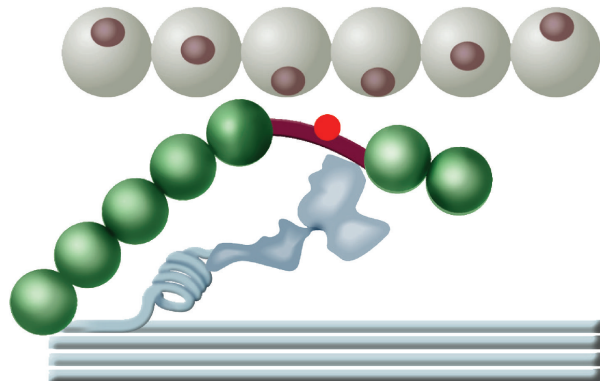

Relaxed state, myosin detached, twitchin detatched

(a)

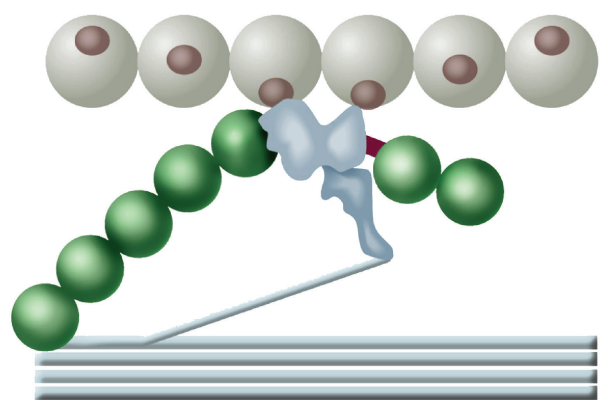

High force state, myosin attached, twitchin detatched

(c)

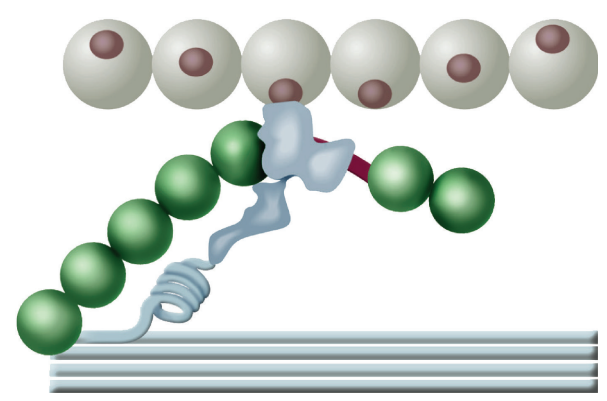

Prepower stroke state, myosin attached, twitchin detatched

(b)

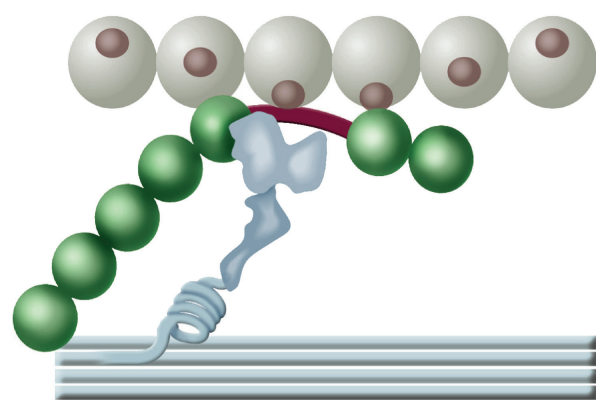

Catch state, myosin attached, twitchin detatched

(d)

FIGURE 13: A model showing twitchin interaction with thick and thin filaments during the myosin crossbridge cycle. (a) The relaxed state. The myosin crossbridge is in the low-force state and detached from the thin filament. The regulatory sites in twitchin are phosphorylated, and twitchin is detached from the thin filament. (b) The prepower stroke state. The myosin crossbridge has been activated by an increase in $\left[\mathrm{Ca}^{+2}\right]$ and the crossbridge binds to the thin filament. Twitchin has been displaced from the thin filament as a result of crossbridge binding to the thin filament. Twitchin is also dephosphorylated by activation of the phosphatase calcineurin. (c) The high-force state. Myosin has gone through the power stroke resulting in force generation. Twitchin is detached from the thin filament by myosin interaction with the thin filament. (d) The catch state. Myosin is in the low-force state and detached from the thin filament. Unphosphorylated twitchin is attached to both the thick and thin filaments. Phosphorylation of twitchin would result in detachment of twitchin from the thin filament and lead to the relaxed state (a). The myosin-twitchin-thin filament interactions described apply independently to both the N-and C-terminal regions of twitchin as shown in the "belt and suspenders" cartoon in Figure 12.

(4) Protein kinase A phosphorylates twitchin to the extent of 3 mole phosphate per mole of twitchin. The sites of phosphorylation, D1 and DX, near the $\mathrm{N}$-terminus, and D2, near the C-terminus have been identified.

(5) Catch occurs in submaximally activated permeabilized ABRM, but not in muscles that are maximally activated with high calcium. On phosphorylation of twitchin, force declines at all submaximal calcium concentrations, with no associated change in ATPase activity. Therefore, catch force maintenance can occur together with cycling myosin crossbridges at submaximal calcium concentrations. Additionally, the catch link can adjust during shortening of submaximally activated muscles and maintain force at the new shorter muscle length.

(6) The twitchin molecule is the catch link that tethers thick and thin filaments. Twitchin binds to both thick and thin filaments, but binding to the latter is phosphorylation dependent. Both the $\mathrm{N}$ - and Cterminal portions of twitchin contain phosphorylation sites that regulate catch, and both of these regions have properties consistent with twitchin being an independent tether between thick and thin filaments.

(7) A model for the mechanism of regulation of catch via a twitchin tether is based on both (a) the phosphorylation state of twitchin and (b) the attachment of myosin crossbridges to actin. When the catch muscle is stimulated, the increase in intracellular calcium is sufficient to maximally activate crossbridges and twitchin is dephosphorylated; the binding of myosin crossbridges to actin displaces twitchin from the thin filament and myosin cyclically interacts with actin with force development and shortening. As the intracellular calcium concentration wanes and catch ensues, the myosin crossbridges detach from actin and the trimeric complex between myosin, twitchin, and actin tethers the thick and thin filaments. The 
muscle relaxes from catch when twitchin is phosphorylated, and the twitchin tether detaches from the thin filament.

(8) The catch state of invertebrate smooth muscle bears many similarities to the tonic force-maintaining state of vertebrate smooth muscles, but represents the extreme condition in terms of economy, largely through the use of an accessory protein (twitchin) to maintain force for extended periods of time. The operation of a similar mechanism in vertebrate smooth muscle remains to be determined, and the challenge for the future will be to identify the counterpart of twitchin and its regulation.

\section{Acknowledgments}

The authors' research has been supported by AR042758-14 from the NIH to MJS and to TMB. They thank Susan Mooers and Srinivasa Narayan for their extraordinary dedication and technical expertise. They acknowledge their colleagues Drs. Betty M. Twarog and Carolyn Cohen, whose research inspired their interest in catch.

\section{References}

[1] J. von Uexkull, "Studien uber den tonus VI. Die pilgermuschel," Zeitschrift für Biologie, vol. 58, pp. 305-322, 1919.

[2] V. N. Kalabokis, E. O’Neall-Hennessey, and A. G. SzentGyorgyi, "Regulatory domains of myosins: influence of heavy chain on $\mathrm{Ca}^{2+}$-binding," Journal of Muscle Research and Cell Motility, vol. 15, no. 5, pp. 547-553, 1994.

[3] N. Ishii, A. W. M. Simpson, and C. C. Ashley, "Free calcium at rest during "catch" in single smooth muscle cells," Science, vol. 243, no. 4896, pp. 1367-1368, 1989.

[4] F. Baguet and J. M. Gillis, "Energy cost of tonic contraction in lamellibranch catch muscle," The Journal of Physiology, vol. 198, pp. 127-143, 1968.

[5] K. M. Nauss and R. E. Davies, "Changes in inorganic phosphate \& arginine during the development, maintenance and loss of tension in the anterior byssus retractor muscle of Mytilus edulis," Biochemische Zeitschrift, vol. 345, pp. 173187, 1966.

[6] R. A. Cole and B. M. Twarog, "Relaxation of catch in a molluscan smooth muscle. I. Effects of drugs which act on the adenyl cyclase system," Comparative Biochemistry and Physiology, vol. 43, no. 2, pp. 321-330, 1972.

[7] R. K. Achazi, B. Doelling, and R. Haakshorst, " 5 Ht induced relaxation \& cyclic AMP in a molluscan smooth muscle," Pflugers Archiv, vol. 349, no. 1, pp. 19-27, 1974.

[8] T. M. Butler, S. U. Mooers, C. Li, S. Narayan, and M. J. Siegman, "Regulation of catch muscle by twitchin phosphorylation: effects on force, ATPase, and shortening," Biophysical Journal, vol. 75, no. 4, pp. 1904-1914, 1998.

[9] J. C. Ruegg, "Smooth muscle tone.," Physiological Reviews, vol. 51, no. 1, pp. 201-248, 1971.

[10] J. Lowy, B. M. Millman, and J. Hanson, "Structure and function in smooth tonic muscles of lammelibranch molluscs," Proceedings of the Royal Society of London. Series B, vol. 160, pp. 525-536, 1964.
[11] J. C. Ruegg, "On the tropomyosin-paramyosin system in relation to the viscous tone of lammellibranch "catch" muscle," Proceedings of the Royal Society of London. Series B, vol. 154, pp. 224-249, 1961.

[12] S. L. Hooper, K. H. Hobbs, and J. B. Thuma, "Invertebrate muscles: thin and thick filament structure; molecular basis of contraction and its regulation, catch and asynchronous muscle," Progress in Neurobiology, vol. 86, no. 2, pp. 72-127, 2008.

[13] C. Cohen, "Matching molecules in the catch mechanism," Proceedings of the National Academy of Sciences of the United States of America, vol. 79, no. 10, pp. 3176-3178, 1982.

[14] N. Pante, "Paramyosin polarity in the thick filament of molluscan smooth muscles," Journal of Structural Biology, vol. 113, no. 2, pp. 148-163, 1994.

[15] R. J. C. Levine, M. Elfvin, M. M. Dewey, and B. Walcott, "Paramyosin in invertebrate muscles. II. Content in relation to structure and function," Journal of Cell Biology, vol. 71, no. 1, pp. 273-279, 1976.

[16] B. R. Jewell, "The nature of the phasic and the tonic responses of the anterior byssal retractor muscle of Mytilus," The Journal of Physiology, vol. 149, pp. 154-177, 1959.

[17] N. S. Shelud'ko, K. F. Tuturova, T. V. Permyakova, S. V. Plotnikov, and A. A. Orlova, "A novel thick filament protein in smooth muscles of bivalve molluscs," Comparative Biochemistry and Physiology B, vol. 122, no. 3, pp. 277-285, 1999.

[18] A. Yamada, M. Yoshio, K. Oiwa, and L. Nyitray, "Catchin, a novel protein in molluscan catch muscles, is produced by alternative splicing from the myosin heavy chain gene," Journal of Molecular Biology, vol. 295, no. 2, pp. 169-178, 2000.

[19] A. Yamada, M. Yoshio, H. Kojima, and K. Oiwa, "An in vitro assay reveals essential protein components for the "catch" state of invertebrate smooth muscle," Proceedings of the National Academy of Sciences of the United States of America, vol. 98, no. 12, pp. 6635-6640, 2001.

[20] R. Hauck and R. K. Achazi, "The ultrastructure of a molluscan catch muscle during a contraction catch relaxation cycle," European Journal of Cell Biology, vol. 45, pp. 30-35, 1987.

[21] P. M. Bennett and A. Elliott, "The "catch" mechanism in molluscan muscle: an electron microscopy study of freezesubstituted anterior byssus retractor muscle of Mytilus edulis," Journal of Muscle Research and Cell Motility, vol. 10, no. 4, pp. 297-311, 1989.

[22] I. Takahashi, M. Shimada, T. Akimoto, T. Kishi, and H. Sugi, "Electron microscopic evidence for the thick filament interconnections associated with the catch state in the anterior byssal retractor muscle of Mytilus edulis," Comparative Biochemistry and Physiology A, vol. 134, no. 1, pp. 115-120, 2003.

[23] A. G. Szent-Gyorgyi, C. Cohen, and J. Kendrick-Jones, "Paramyosin and the filaments of molluscan "catch" muscles. II. Native filaments: Isolation and characterization," Journal of Molecular Biology, vol. 56, no. 2, pp. 239-248, 1971.

[24] H. F. Epstein, B. J. Aronow, and H. E. Harris, "Myosin paramyosin cofilaments: enzymatic interaction with F-actin," Proceedings of the National Academy of Sciences of the United States of America, vol. 73, no. 9, pp. 3015-3019, 1976.

[25] J. C. Ruegg, "Myosin-linked regulation of molluscan muscle," in Calcium in Muscle Contraction, Springer, New York, NY, USA, 2nd edition, 1992. 
[26] R. K. Achazi, "Phosphorylation of molluscan paramyosin," Pflugers Archiv European, vol. 379, no. 2, pp. 197-201, 1979.

[27] R. K. Achazi, "The catch muscle," in Basic Biology of Muscle: A Comparative Approach, pp. 291-308, Plenum, New York, NY, USA, 1982.

[28] B. Ljung and P. Hallgren, "On the mechanism of inhibitory action of vibratins as studied in a molluscan catch muscle and in vertebrate vascular smooth muscle," Acta Physiologica Scandinavica, vol. 95, no. 4, pp. 424-430, 1975.

[29] B. Ljung and R. Sivertsson, "Vibration induced inhibition of vascular smooth muscle contraction," Blood Vessels, vol. 12, no. 1, pp. 38-52, 1975.

[30] T. Kobayashi, C. Ichikawa, and H. Sugi, "Differential effects of sinusoidal vibrations on tension and stiffness in Mytilus smooth muscle during catch state.," Japanese Journal of Physiology, vol. 35, no. 4, pp. 689-692, 1985.

[31] J. Lowy and B. M. Millman, "The contractile mechanism of the anterior byssus retractor of Mytilus edulis," Proceedings of the Royal Society of London. Series B, vol. 246, pp. 105-148, 1963.

[32] G. Pfitzer and J. C. Ruegg, "Molluscan catch muscle: regulation and mechanics in living and skinned anterior byssus retractor muscle of Mytilus edulis," Journal of Comparative Physiology, vol. 147, no. 1, pp. 137-142, 1982.

[33] W. H. Johnson and B. M. Twarog, "The basis for prolonged contractions in molluscan muscles.", The Journal of General Physiology, vol. 43, pp. 941-960, 1960.

[34] M. J. Kushmerick and R. E. Davies, "The chemical energetics of muscle contraction. II. The chemistry, efficiency and power of maximally working sartorius muscles," Proceedings of the Royal Society of London. Series B, vol. 174, no. 36, pp. 315-353, 1969.

[35] B. M. Twarog, "The regulation of catch in molluscan muscle," Journal of General Physiology, vol. 50, no. 6, pp. 157-169, 1967.

[36] E. Bozler, "The heat production of smooth muscle," The Journal of Physiology, vol. 69, pp. 442-462, 1930.

[37] T. Tameyasu and H. Sugi, "The series elastic component and the force-velocity relation in the anterior byssal retractor muscle of Mytilus edulis during active and catch contractions," Journal of Experimental Biology, vol. 64, no. 2, pp. 497510, 1976.

[38] M. J. Siegman, T. M. Butler, S. U. Mooers, and R. E. Davies, "Chemical energetics of force development, force maintenance, and relaxation in mammalian smooth muscle," Journal of General Physiology, vol. 76, no. 5, pp. 609-629, 1980.

[39] P. F. Dillon, M. O. Aksoy, S. P. Driska, and R. A. Murphy, "Myosin phosphorylation and crossbridge cycle in arterial smooth muscle," Science, vol. 211, pp. 495-497, 1981.

[40] A. P. Somlyo and A. V. Somlyo, "Discussion," The Journal of General Physiology, vol. 50, pp. 168-169, 1967.

[41] J. Kendrick-Jones, W. Lehman, and A. G. Szent-Györgyi, "Regulation in molluscan muscles," Journal of Molecular Biology, vol. 54, no. 2, pp. 313-326, 1970.

[42] F. Baguet, "The catch-state in glycerol extracted fibres from a lamellibranch smooth muscle," European Journal of Physiology, vol. 340, no. 1, pp. 19-34, 1973.

[43] G. Marchand Dumont and F. Baguet, "The control mechanism of relaxation in molluscan catch muscle (ABRM)," Pflugers Archiv, vol. 354, no. 1, pp. 87-100, 1975.

[44] F. Cornelius, "The regulation of tension in a chemically skinned molluscan smooth muscle. Effect of $\mathrm{Mg}^{2+}$ on the $\mathrm{Ca}^{2+}$-activated tension generation," Journal of General Physiology, vol. 75, no. 6, pp. 709-725, 1980.

[45] F. Cornelius, "Tonic contraction and the control of relaxation in a chemically skinned molluscan smooth muscle," Journal of General Physiology, vol. 79, no. 5, pp. 821-834, 1982.

[46] L. Castellani and C. Cohen, "Myosin rod phosphorylation and the catch state of molluscan-muscles," Science, vol. 235, no. 4786, pp. 334-337, 1987.

[47] K. Guth, M. Gagelmann, and J. C. Ruegg, "Skinned smooth muscle: time course of force and ATPase activity during contraction cycle," Experientia, vol. 40, no. 2, pp. 174-176, 1984.

[48] M. Schneider, M. Sparrow, and J. C. Rueegg, "Inorganic phosphate promotes relaxation of chemically skinned smooth muscle of guinea-pig Taenia coli," Experientia, vol. 37, no. 9, pp. 980-982, 1981.

[49] S. Watabe and D. J. Hartshorne, "Paramyosin and the catch mechanism," Comparative Biochemistry and Physiology, vol. 96, no. 4, pp. 639-646, 1990.

[50] S. Watabe, T. Tsuchiya, and D. J. Hartshorne, "Phosphorylation of paramyosin," Comparative Biochemistry and Physiology B, vol. 94, no. 4, pp. 813-821, 1989.

[51] H. Sohma, M. Yazawa, and F. Morita, "Phosphorylation of regulatory light chain a (RLC-a) in smooth muscle myosin of scallop, Patinopecten yessoensis," Journal of Biochemistry, vol. 98 , no. 2, pp. 569-572, 1985.

[52] H. Sohma and F. Morita, "Purification of a protein kinase phosphorylating myosin regulatory light chain-a (RLC-a) from smooth muscle of scallop, Patinopecten yessoensis," Journal of Biochemistry, vol. 100, no. 5, pp. 1155-1163, 1986.

[53] H. Sohma, H. Sasada, K. Inoue, and F. Morita, "Regulatory light chain-a myosin kinase (aMK) catalyzes phosphorylation of smooth muscle myosin heavy chains of scallop, Patinopecten yessoensis," Journal of Biochemistry, vol. 104, no. 6, pp. 889-893, 1988.

[54] L. Castellani and C. Cohen, "Rod phosphorylation favors folding in a catch muscle myosin," Proceedings of the National Academy of Sciences of the United States of America, vol. 84, no. 12, pp. 4058-4062, 1987.

[55] L. Castellani, B. W. Elliott Jr., and C. Cohen, "Phosphorylatable serine residues are located in a non-helical tailpiece of a catch muscle myosin," Journal of Muscle Research and Cell Motility, vol. 9, no. 6, pp. 533-540, 1988.

[56] L. Castellani and C. Cohen, "A calcineurin-like phosphatase is required for catch contraction," FEBS Letters, vol. 309, no. 3, pp. 321-326, 1992.

[57] R. Hauck and R. K. Achazi, "In situ phosphorylation of contractile proteins of a molluscan (Mytilus edulis) catch muscle in different functional states," Comparative Biochemistry and Physiology B, vol. 100, no. 2, pp. 237-242, 1991.

[58] M. J. Siegman, S. U. Mooers, C. Li, et al., "Phosphorylation of a high molecular weight (approximately $600 \mathrm{kDa}$ ) protein regulates catch in invertebrate smooth muscle," Journal of Muscle Research and Cell Motility, vol. 18, no. 6, pp. 655-670, 1997.

[59] R. H. Waterston, J. N. Thomson, and S. Brenner, "Mutants with altered muscle structure in Caenorhabditis elegans," Developmental Biology, vol. 77, no. 2, pp. 271-302, 1980.

[60] D. G. Moerman, G. M. Benian, R. J. Barstead, L. A. Schriefer, and R. H. Waterston, "Identification and intracellular localization of the unc-22 gene product of Caenorhabditis elegans," Genes \& Development, vol. 2, no. 1, pp. 93-105, 1988. 
[61] P. Vibert, S. M. Edelstein, L. Castellani, and B. W. Elliott Jr., "Mini-titins in striated and smooth molluscan muscles: structure, location and immunological crossreactivity," Journal of Muscle Research and Cell Motility, vol. 14, no. 6, pp. 598-607, 1993.

[62] W. C. Probst, E. C. Cropper, J. Heierhorst, et al., "cAMPdependent phosphorylation of Aplysia twitchin may mediate modulation of muscle contractions by neuropeptide cotransmitters," Proceedings of the National Academy of Sciences of the United States of America, vol. 91, no. 18, pp. 8487-8491, 1994.

[63] M. J. Siegman, D. Funabara, S. Kinoshita, S. Watabe, D. J. Hartshorne, and T. M. Butler, "Phosphorylation of a twitchin-related protein controls catch and calcium sensitivity of force production in invertebrate smooth muscle," Proceedings of the National Academy of Sciences of the United States of America, vol. 95, no. 9, pp. 5383-5388, 1998.

[64] D. Funabara, S. Watabe, S. U. Mooers, et al., "Twitchin from molluscan catch muscle. Primary structure and relationship between site-specific phosphorylation and mechanical function," Journal of Biological Chemistry, vol. 278, no. 31, pp. 29308-29316, 2003.

[65] J. E. Kiff, D. G. Moerman, L. A. Schriefer, and R. H. Waterston, "Transposon-induced deletions in unc-22 of $C$. elegans associated with almost normal gene activity," Nature, vol. 331, no. 6157, pp. 631-633, 1988.

[66] B. Bullard, W. A. Linke, and K. Leonard, "Varieties of elastic protein in invertebrate muscles," Journal of Muscle Research and Cell Motility, vol. 23, no. 5-6, pp. 435-447, 2002.

[67] R. Southgate and A. Ayme-Southgate, "Alternative splicing of an amino-terminal PEVK-like region generates multiple isoforms of Drosophila projectin," Journal of Molecular Biology, vol. 313, no. 5, pp. 1035-1043, 2001.

[68] T. Oshino, J. Shimamura, A. Fukuzawa, K. Maruyama, and S. Kimura, "The entire cDNA sequences of projectin isoforms of crayfish claw closer and flexor muscles and their localization," Journal of Muscle Research and Cell Motility, vol. 24, no. 7, pp. 431-438, 2003.

[69] G. M. Benian, S. W. L'Hernault, and M. E. Morris, "Additional sequence complexity in the muscle gene, unc-22, and its encoded protein, twitchin, of Caenorhabditis elegans," Genetics, vol. 134, no. 4, pp. 1097-1104, 1993.

[70] P.-J. Lin, K. Luby-Phelps, and J. T. Stull, "Properties of filament-bound myosin light chain kinase," Journal of Biological Chemistry, vol. 274, no. 9, pp. 5987-5994, 1999.

[71] L. Smith, M. Parizi-Robinson, M.-S. Zhu, et al., "Properties of long myosin light chain kinase binding to F-actin in vitro and in vivo," Journal of Biological Chemistry, vol. 277, no. 38, pp. 35597-35604, 2002.

[72] P.-J. Lin, K. Luby-Phelps, and J. T. Stull, "Binding of myosin light chain kinase to cellular actin-myosin filaments," Journal of Biological Chemistry, vol. 272, no. 11, pp. 7412-7420, 1997.

[73] L. Smith, X. Su, P.-J. Lin, G. Zhi, and J. T. Stull, "Identification of a novel actin binding motif in smooth muscle myosin light chain kinase," Journal of Biological Chemistry, vol. 274, no. 41, pp. 29433-29438, 1999.

[74] L. Smith and J. T. Stull, "Myosin light chain kinase binding to actin filaments," FEBS Letters, vol. 480, no. 2-3, pp. 298-300, 2000.

[75] V. Hatch, G. Zhi, L. Smith, J. T. Stull, R. Craig, and W. Lehman, "Myosin light chain kinase binding to a unique site on $\mathrm{F}$-actin revealed by three-dimensional image reconstruction," Journal of Cell Biology, vol. 154, no. 3, pp. 611-617, 2001.
[76] W. A. Linke, M. Kulke, H. Li, et al., "PEVK domain of titin: an entropic spring with actin-binding properties," Journal of Structural Biology, vol. 137, no. 1-2, pp. 194-205, 2002.

[77] M. Kusaka, D. Ikeda, D. Funabara, D. J. Hartshorne, and S. Watabe, "The occurrence of tissue-specific twitchin isoforms in the mussel Mytilus galloprovincialis," Fisheries Science, vol. 74, no. 3, pp. 677-686, 2008.

[78] A. S. Franke, S. U. Mooers, S. R. Narayan, M. J. Siegman, and T. M. Butler, "Myosin cross-bridge kinetics and the mechanism of catch," Biophysical Journal, vol. 93, no. 2, pp. 554-565, 2007.

[79] W. O. Fenn, "A quantitative comparison between the energy liberated and the work performed by the isolated sartorius muscle of the frog," The Journal of Physiology, vol. 58, pp. 175-203, 1923.

[80] A. F. Huxley, "Muscle structure and theories of contraction," Progress in Biophysics and Biophysical Chemistry, vol. 7, pp. 255-318, 1957.

[81] M. Nyitrai and M. A. Geeves, "Adenosine diphosphate and strain sensitivity in myosin motors," Philosophical Transactions of the Royal Society B, vol. 359, no. 1452, pp. 1867-1877, 2004.

[82] T. M. Butler, S. R. Narayan, S. U. Mooers, and M. J. Siegman, "Strain dependence of crossbridge kinetics in smooth muscle," Biophysical Journal, vol. 68, p. A169, 1995.

[83] A. S. Khromov, M. R. Webb, M. A. Ferenczi, D. R. Trentham, A. P. Somlyo, and A. V. Somlyo, "Myosin regulatory light chain phosphorylation and strain modulate adenosine diphosphate release from smooth muscle myosin," Biophysical Journal, vol. 86, no. 4, pp. 2318-2328, 2004.

[84] T. M. Butler, S. R. Narayan, S. U. Mooers, D. J. Hartshorne, and M. J. Siegman, "The myosin cross-bridge cycle and its control by twitchin phosphorylation in catch muscle," Biophysical Journal, vol. 80, no. 1, pp. 415-426, 2001.

[85] A. V. Somlyo, Y. E. Goldman, T. Fujimori, M. Bond, D. R. Trentham, and A. P. Somlyo, "Cross-bridge kinetics, cooperativity, and negatively strained cross-bridges in vertebrate smooth muscle. A laser-flash photolysis study," Journal of General Physiology, vol. 91, no. 2, pp. 165-192, 1988.

[86] C.-M. Hai and R. A. Murphy, "Cross-bridge phosphorylation and regulation of latch state in smooth muscle," American Journal of Physiology, vol. 254, no. 1, pp. C99-C106, 1988.

[87] T. B. Vyas, S. U. Mooers, S. R. Narayan, J. C. Witherell, M. J. Siegman, and T. M. Butler, "Cooperative activation of myosin by light chain phosphorylation in permeabilized smooth muscle," American Journal of Physiology, vol. 263, no. 1, pp. C210-C219, 1992.

[88] T. B. Vyas, S. U. Mooers, S. R. Narayan, M. J. Siegman, and T. M. Butler, "Cross-bridge cycling at rest and during activation: turnover of myosin- bound ADP in permeabilized smooth muscle," Journal of Biological Chemistry, vol. 269, no. 10, pp. 7316-7322, 1994.

[89] C. M. Rembold, R. L. Wardle, C. J. Wingard, T. W. Batts, E. F. Etter, and R. A. Murphy, "Cooperative attachment of cross bridges predicts regulation of smooth muscle force by myosin phosphorylation," American Journal of PhysiologyCell Physiology, vol. 287, no. 3, pp. C594-C602, 2004.

[90] P. A. Ellison, J. R. Sellers, and C. R. Cremo, "Kinetics of smooth muscle heavy meromyosin with one thiophosphorylated head," Journal of Biological Chemistry, vol. 275, no. 20, pp. 15142-15151, 2000.

[91] H. Tanaka, K. Homma, H. D. White, T. Yanagida, and M. Ikebe, "Smooth muscle myosin phosphorylated at single 
head shows sustained mechanical activity," Journal of Biological Chemistry, vol. 283, no. 23, pp. 15611-15618, 2008.

[92] A. Khromov, A. V. Somlyo, D. R. Trentham, B. Zimmermann, and A. P. Somlyo, "The role of MgADP in force maintenance by dephosphorylated cross-bridges in smooth muscle: a flash photolysis study," Biophysical Journal, vol. 69, no. 6, pp. 26112622, 1995.

[93] S.-N. Yu, P. E. Crago, and H. J. Chiel, "A nonisometric kinetic model for smooth muscle," American Journal of Physiology, vol. 272, no. 3, pp. C1025-C1039, 1997.

[94] A. F. Straight, A. Cheung, J. Limouze, et al., "Dissecting temporal and spatial control of cytokinesis with a myosin II inhibitor," Science, vol. 299, no. 5613, pp. 1743-1747, 2003.

[95] J. Limouze, A. F. Straight, T. Mitchison, and J. R. Sellers, "Specificity of blebbistatin, hibitor of myosin II," Journal of Muscle Research and Cell Motility, vol. 25, no. 4-5, pp. 337341, 2004.

[96] M. Kovacs, J. Toth, C. Hetenyi, A. Malnasi-Csizmadia, and J. R. Seller, "Mechanism of blebbistatin inhibition of myosin II," Journal of Biological Chemistry, vol. 279, no. 34, pp. 35557-35563, 2004.

[97] J. S. Allingham, R. Smith, and I. Rayment, "The structural basis of blebbistatin inhibition and specificity for myosin II," Nature Structural and Molecular Biology, vol. 12, no. 4, pp. 378-379, 2005.

[98] T. M. Butler, S. U. Mooers, and M. J. Siegman, "Catch force links and the low to high force transition of myosin," Biophysical Journal, vol. 90, no. 9, pp. 3193-3202, 2006.

[99] S. V. Avrova, N. S. Shelud'ko, Y. S. Borovikov, and S. Galler, "Twitchin of mollusc smooth muscles can induce "catch"like properties in human skeletal muscle: support for the assumption that the "catch" state involves twitchin linkages between myofilaments," Journal of Comparative Physiology B, vol. 179, no. 8, pp. 945-950, 2009.

[100] Y. Tsutsui, M. Yoshio, K. Oiwa, and A. Yamada, "Striated muscle twitchin of bivalves has "catchability", the ability to bind thick filaments tightly to thin filaments, representing the catch state," Journal of Molecular Biology, vol. 365, no. 2, pp. 325-332, 2007.

[101] A. Yamada, N. Ishii, T. Shimmen, and K. Takahashi, "MgATPase activity and motility of native thick filaments isolated from the anterior byssus retractor muscle of Mytilus edulis," Journal of Muscle Research and Cell Motility, vol. 10, no. 2, pp. 124-134, 1989.

[102] N. S. Shelud'ko, O. S. Matusovsky, T. V. Permyakova, and G. G. Matusovskaya, “"Twitchin-actin linkage hypothesis" for the catch mechanism in molluscan muscles: evidence that twitchin interacts witch myosin, myorod, and paramyosin core and affects properties of actomyosin," Archives of Biochemistry and Biophysics, vol. 466, pp. 125-135, 2007.

[103] N. S. Shelud'ko, G. G. Matusovskaya, T. V. Permyakova, and O. S. Matusovsky, "Twitchin, a thick-filament protein from molluscan catch muscle, interacts with F-actin in a phosphorylation-dependent way," Archives of Biochemistry and Biophysics, vol. 432, no. 2, pp. 269-277, 2004.

[104] D. Funabara, C. Hamamoto, K. Yamamoto, et al., "Unphosphorylated twitchin forms a complex with actin and myosin that may contribute to tension maintenance in catch," Journal of Experimental Biology, vol. 210, no. 24, pp. 4399-4410, 2007.

[105] D. Funabara, R. Osawa, M. Ueda, S. Kanoh, D. J. Hartshorne, and S. Watabe, "Myosin loop 2 is involved in the formation of a trimeric complex of twitchin, actin, and myosin," Journal of Biological Chemistry, vol. 284, no. 27, pp. 18015-18020, 2009.

[106] J. F. Shaffer, R. W. Kensler, and S. P. Harris, "The myosin-binding protein $\mathrm{C}$ motif binds to F-actin in a phosphorylation-sensitive manner," Journal of Biological Chemistry, vol. 284, no. 18, pp. 12318-12327, 2009.

[107] M. Gruen and M. Gautel, "Mutations in $\beta$-myosin S2 that cause familial hypertrophic cardiomyopathy (FHC) abolish the interaction with the regulatory domain of myosinbinding protein-C," Journal of Molecular Biology, vol. 286, no. 3, pp. 933-949, 1999.

[108] M. Gruen, H. Prinz, and M. Gautel, "cAPK-phosphorylation controls the interaction of the regulatory domain of cardiac myosin binding protein $\mathrm{C}$ with myosin-S2 in an on-off fashion," FEBS Letters, vol. 453, no. 3, pp. 254-259, 1999. 

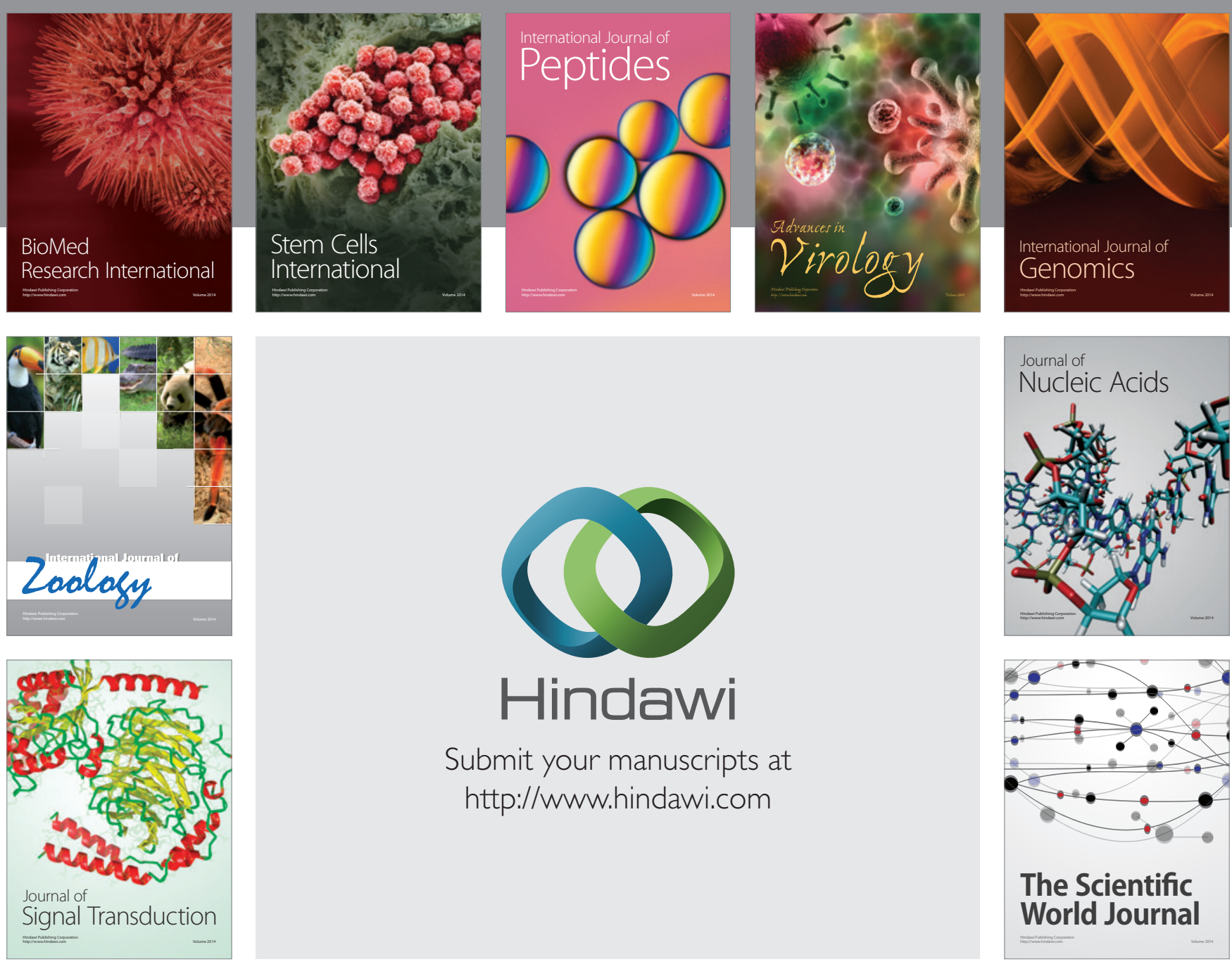

Submit your manuscripts at

http://www.hindawi.com
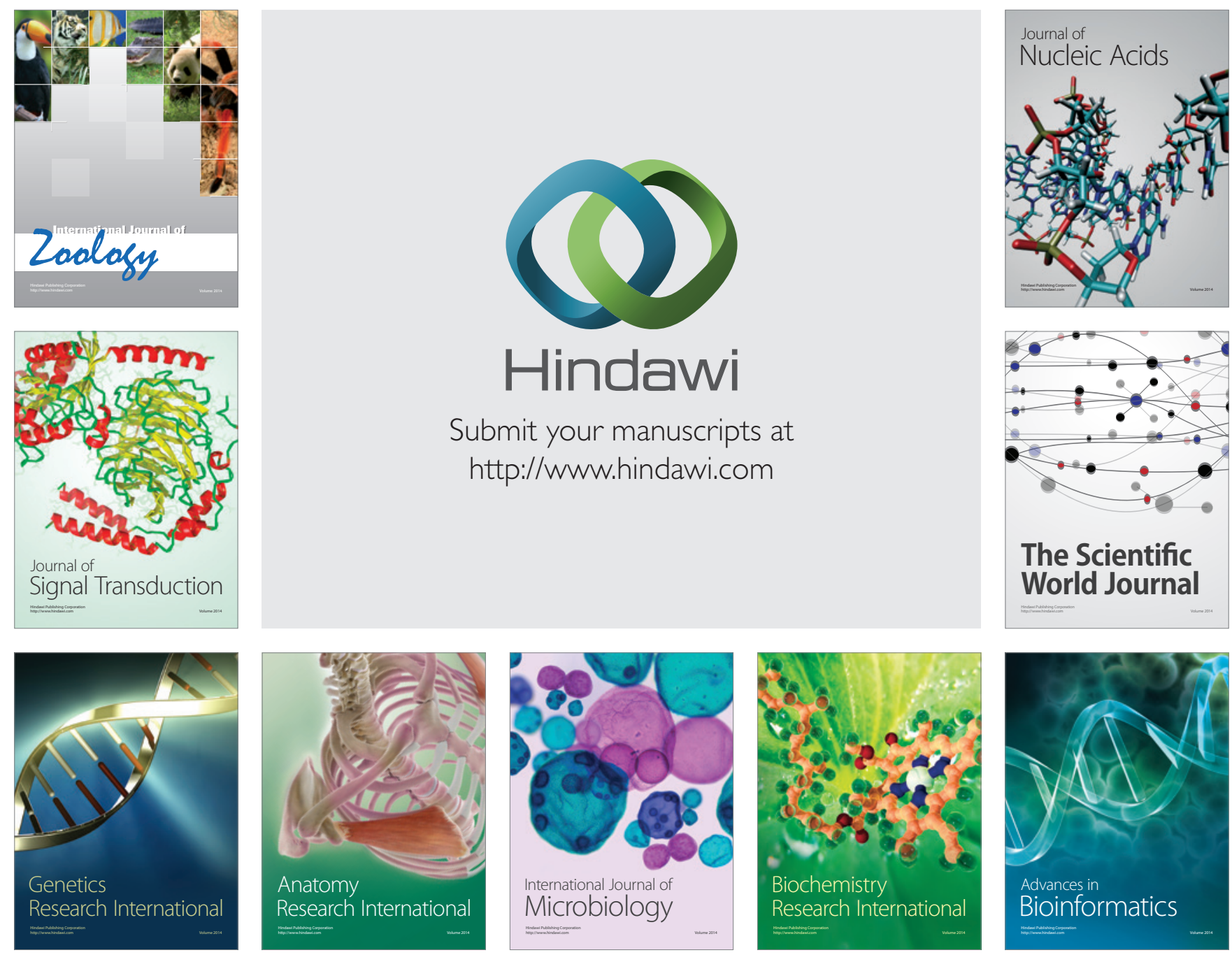

The Scientific World Journal
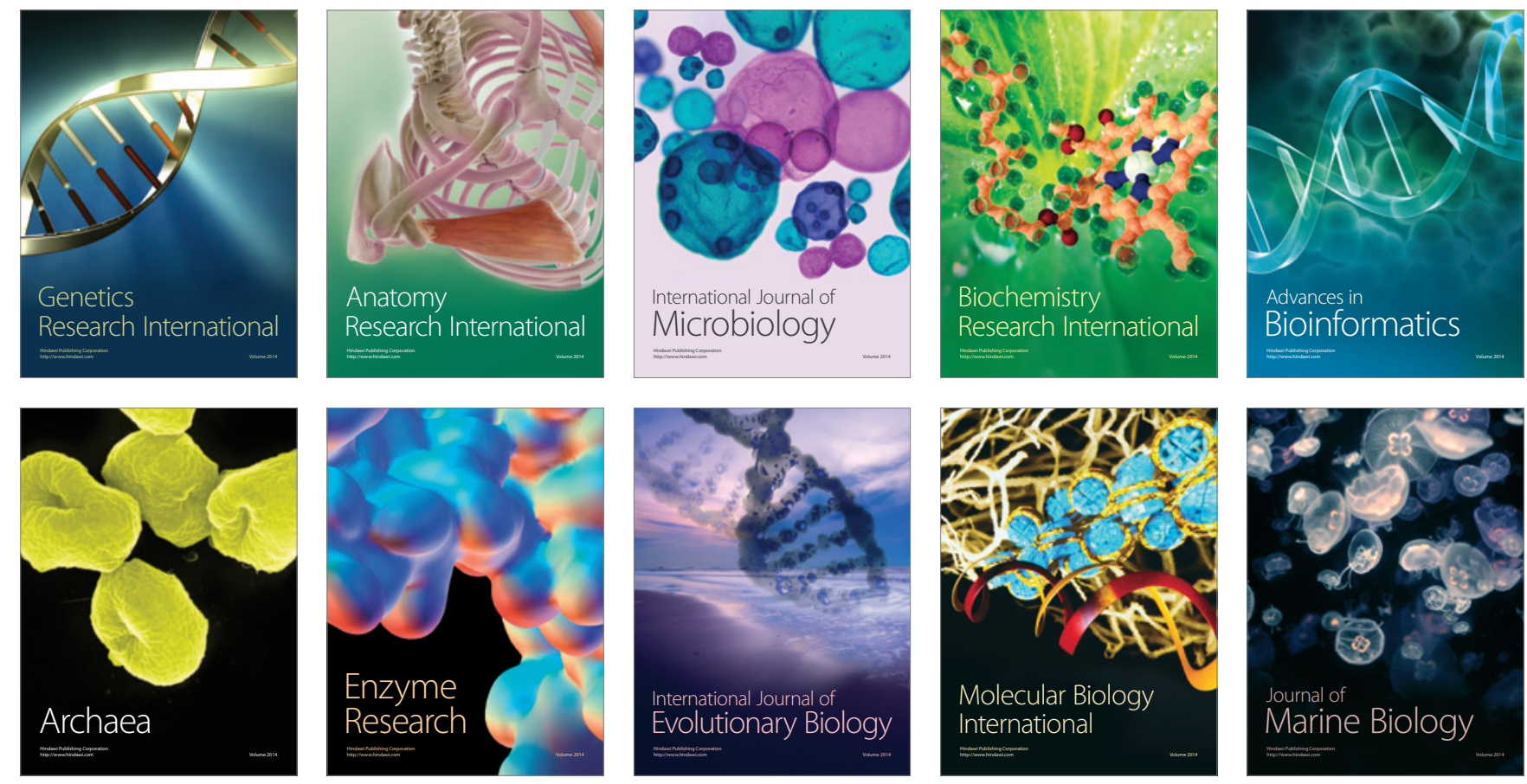